\title{
Spline collocation for convolutional parabolic boundary integral equations
}

\author{
M. Costabel $^{1}$, J. Saranen ${ }^{2}$ \\ 1 Institut de Mathématiques, Université de Rennes I, Campus de Beaulieu, F-35042 Rennes \\ Cedex, France; e-mail: costabel@univ-rennes1.fr \\ 2 Department of Mathematical Sciences, University of Oulu, FIN-90570 Oulu, Finland; \\ e-mail: jukka.saranen@oulu.fi
}

Received December 15, 1997 / Revised version received November 16, 1998 / Published online September 24, 1999

Summary. We consider spline collocation methods for a class of parabolic pseudodifferential operators. We show optimal order convergence results in a large scale of anisotropic Sobolev spaces. The results cover for example the case of the single layer heat operator equation when the spatial domain is a disc.

Mathematics Subject Classification (1991): 65R20, 45L10

\section{Introduction}

The integral equation method for the solution of parabolic problems is known already for a long time, for the early literature see [23, 21, 28, 29, 30]. The reasons which recommend this method instead of the domain methods are similar as in the elliptic case. The main arguments are: In the level of the numerical implementation there is a reduction in the dimension of the matrix equation to be solved. The method is very suitable for exterior problems. Moreover, by using the direct method the unknown function is a quantity of physical interest.

In contrast to the elliptic case, there does not exist any general theory for the numerical solution of the parabolic boundary integral equations. There are results which are limited to special examples. The equations of the second kind have been studied in the works of Onishi [25], and Costabel, Onishi, Wendland [10]. For the equations of the first kind, there are satisfactory

Correspondence to: $\mathrm{M}$. Costabel 
results only for the Galerkin solution of the single layer heat equation [8, 24]. The computationally more attractive collocation method is not yet fully understood. Hamina and Saranen [15] were able to show convergence of the spline collocation for the single layer heat equation in the case of the circle. These results have been extended by Hämäläinen and Saranen $[19,20]$ to the case of a general domain with smooth boundary when the arclength is used as parametrization. However, the convergence results which they obtained are not of optimal order. For a different approach using time discretization for parabolic boundary integral equations we refer to [22].

In the study of the numerical methods for parabolic boundary integral equations, it is useful to know which other representations for the operators are available in addition to the natural kernel representation. In the elliptic case, application of the theory of pseudodifferential operators has turned out be very efficient and a large variety of general results have been obtained by this framework. It seems now possible to analyze numerical schemes for parabolic boundary integral equations with the help of parabolic pseudodifferential operators acting in anisotropic Sobolev spaces.

The present paper gives a starting point in this direction; we obtain optimal order convergence results for the spline collocation method for the class of operators which we call of convolutional type. In practical terms this class covers equations given on circular boundaries. In future works we will discuss more general types of operators which cover the case of general smooth boundaries and even moving domains.

It is worth mentioning that even in the case of elliptic problems, the stability and convergence of spline collocation in higher dimensions is not generally known. To our knowledge, there are results only for some special cases, where the equation is given on a torus $[3,32,9]$, square or cube $[11$, 31]. For the spline collocation in the one dimensional case we refer to the basic papers $[4,5,33,34]$. In our case the integral equation is given on a cylindrical domain. For the discretization we use uniform meshes and the analysis of the collocation equations is based mainly on Fourier techniques. However, it was not a priori obvious how to handle efficiently the collocation problem in this situation, where the mesh is infinite in one direction.

For the numerical solution, one considers a problem on a finite time interval, which leads to the solution of a finite-dimensional system of equations. For the numerical analysis, however, it turned out that one cannot treat this finite-dimensional problem directly, so we had to choose a different way. The basic idea of our approach is to consider first the "whole space problem", i. e. the problem with the infinite time axis, for a properly chosen principal part of the operator. For the solution of this whole space problem, we apply discrete Fourier transforms in both the space and the time direction. With respect to the space variable, this is the conventional discrete Fourier 
transform method for periodic functions (or sequences). With respect to the time variable, this transform is a periodic function which is defined for a class of non-periodic functions (sequences) on the whole time interval. Now the discretized whole-space problem is an infinite-dimensional system of equations which requires different tools from those used traditionally.

One of the main properties which makes the analysis of the collocation method possible is the coercivity of the parabolic boundary integral operators in an anisotropic Sobolev space. The discrete analogue of this property, namely the ellipticity of the numerical symbol, implies the stability of the method. This ellipticity requires a complicated proof in the case of splines of even degree (see the Appendix). A similar situation is known from the analysis of the higher dimensional spline collocation for elliptic equations, see Costabel and McLean [9]. For the stability and optimal order estimates we need to impose the well-known condition relating the space and time step sizes, $h_{t} \sim h_{\theta}^{2}=h^{2}$. Although we have focused here on parabolic boundary integral equations, it seems that our method may be useful for the analysis of elliptic problems in the non-periodic case, too.

In addition to the basic optimal order convergence results, we obtain improved error estimates when using even degree splines in the space direction. These results extend the observation of Saranen [33] to the parabolic case. They are useful in particular when heat potentials are approximated by the collocation method for boundary densities. The basic examples covered by our work are the single layer and the hypersingular heat operators. In order to preserve the vanishing initial condition of the heat equation, we have to use splines of low degree in the time direction: only piecewise constant or piecewise linear continuous splines are considered. With this restriction of the order of the method, we obtain convergence of order $O\left(h^{5}\right)$ for the single layer operator and $O\left(h^{3}\right)$ for the hypersingular heat operator.

\section{Preliminaries}

In the following analysis we use anisotropic Sobolev spaces of functions (distributions) $u(\theta, t)$ which are 1-periodic with respect to the spatial variable $\theta$. For $s \in \mathbb{R}$ let $H^{s, \frac{s}{2}}=H^{s, \frac{s}{2}}\left(\mathbb{R}_{\theta} \times \mathbb{R}_{t}\right)$ to be the anisotropic space with the norm $\|u\|_{s, \frac{s}{2}}$ given by

$$
\|u\|_{s, \frac{s}{2}}^{2}=\frac{1}{2 \pi} \sum_{n \in \mathbb{Z}} \int_{\mathbb{R}}\left(1+|n|+|\eta|^{\frac{1}{2}}\right)^{2 s}|\hat{u}(n, \eta)|^{2} d \eta,
$$


where

$$
\hat{u}(n, \eta)=\int_{-\frac{1}{2}}^{\frac{1}{2}} \int_{\mathbb{R}} \mathrm{e}^{-\mathrm{i} n 2 \pi \theta-\mathrm{i} \eta t} u(\theta, t) d t d \theta .
$$

We also need the corresponding spaces of functions, where the vanishing initial condition at $t=0$ and finite time interval are taken into account. For this we put $\mathbb{R}_{T}^{2}:=\mathbb{R}_{\theta} \times(0, T)$ and define

$$
\begin{aligned}
\tilde{H}^{s, \frac{s}{2}} & =\left\{u \in H^{s, \frac{s}{2}} \mid \operatorname{supp} u \subset \mathbb{R}_{\theta} \times[0, \infty)\right\}, \\
\tilde{H}^{s, \frac{s}{2}}\left(\mathbb{R}_{T}^{2}\right) & =\left\{u=\left.U\right|_{\mathbb{R}_{\theta} \times(-\infty, T)}: U \in \tilde{H}^{s, \frac{s}{2}}\right\} .
\end{aligned}
$$

The norm of the spaces $\tilde{H}^{s, \frac{s}{2}}\left(\mathbb{R}_{T}^{2}\right)$ is given by the usual infimum norm,

$$
\|\left. u\right|_{s, \frac{s}{2} ; T}=\inf \left\{\|U\|_{s, \frac{s}{2}}: u=\left.U\right|_{\mathbb{R}_{\theta} \times(-\infty, T)}, U \in \tilde{H}^{s, \frac{s}{2}}\right\} .
$$

In the following we consider operators which are of Volterra type. By the definition the operator $L$ is of Volterra type, if the following property is satisfied for all $t \in \mathbb{R}$ : if $u$ vanishes in the domain $\tau<t$, then $L u$ also has this property. We assume that the operator $L$ takes the form

$$
L=A+B,
$$

where the main part $A$ is given by

$$
A u(\theta, t)=\frac{1}{2 \pi} \sum_{n \in \mathbb{Z}} \int_{\mathbb{R}} a(n, \eta) \hat{u}(n, \eta) \mathrm{e}^{\mathrm{i} n 2 \pi \theta+\mathrm{i} \eta t} d \eta
$$

and the symbol $a(\xi, \eta)$ satisfies the following conditions with $\beta \in \mathbb{R}, c_{0}>$ 0 , and a number $0<\gamma \leq 1$,

(2.1c) $a \in C^{\infty}\left(\mathbb{R}^{2}\right)$,

(2.1d) $a\left(\lambda \xi, \lambda^{2} \eta\right)=\lambda^{\beta} a(\xi, \eta), \lambda \geq 1,|\xi|+|\eta|^{\frac{1}{2}} \geq \gamma$,

(2.1e) $\operatorname{Re} a(\xi, \eta) \geq c_{0}\left(|\xi|+|\eta|^{\frac{1}{2}}\right)^{\beta}, \quad|\xi|+|\eta|^{\frac{1}{2}} \geq \gamma$,

(2.1f) The mapping $\eta \mapsto a(\xi, \eta)$ has a polynomially bounded analytic continuation

into the domain $z=\eta-\mathrm{i} \sigma, \sigma>0$ which is continuous for $\sigma \geq 0$,

(2.1g) $B: \tilde{H}^{s, \frac{s}{2}}\left(\mathbb{R}_{T}^{2}\right) \rightarrow \tilde{H}^{s-\beta+\delta, \frac{s-\beta+\delta}{2}}\left(\mathbb{R}_{T}^{2}\right)$ is bounded for a $0<\delta \leq 1$ the operator $B$ is of Volterra type,

(2.1h) $L: \tilde{H}^{s, \frac{s}{2}}\left(\mathbb{R}_{T}^{2}\right) \rightarrow \tilde{H}^{s-\beta, \frac{s-\beta}{2}}\left(\mathbb{R}_{T}^{2}\right)$ is an isomorphism.

Remark 2.1 The function $a(\xi, \eta),|\xi|+|\eta|^{\frac{1}{2}} \geq \gamma$ is the called principal symbol of the operator $L$. Note that (2.1d) implies the upper bound

$$
|a(\xi, \eta)| \leq c_{1}\left(|\xi|+|\eta|^{\frac{1}{2}}\right)^{\beta}, \quad|\xi|+|\eta|^{\frac{1}{2}} \geq \gamma .
$$


In the case of the classical parabolic boundary integral operators the principal symbol $a(\xi, \eta)$ is defined for all $(\xi, \eta) \neq(0,0)$ which means that $\gamma$ can be taken to be arbitrarily small. This fact has some importance later when discussing the collocation method.

Remark 2.2 Condition (2.1e) describes the coercivity of the operator. It is essential for the numerical analysis, in particular for the stability of numerical approximations. This property does not appear in the general theory of pseudodifferential operators of Volterra type in [26, 27].

Remark 2.3 Condition (2.1f) describes the Volterra property of $A$ [26].

Remark 2.4 Particular examples of operators which satisfy assumption (2.1) are the single layer heat operator $(\beta=-1)$ and the hypersingular heat operator $(\beta=1)$ when the spatial domain is a disc. For the general domains with smooth boundary (2.1) covers the equations of the second kind. In these cases we may choose $\delta=1$.

From (2.2) it follows that $A$ defines a continuous mapping $A: H^{s, \frac{s}{2}} \rightarrow$ $H^{s-\beta, \frac{s-\beta}{2}}$. Moreover, using the Volterra property we deduce that $A$ : $\tilde{H}^{s, \frac{s}{2}}\left(\mathbb{R}_{T}^{2}\right) \rightarrow \tilde{H}^{s-\beta, \frac{s-\beta}{2}}\left(\mathbb{R}_{T}^{2}\right)$ is well-defined and bounded. For a more general class of operators including those described by the assumption (2.1), see $[26,27]$.

Example 2.1 Let $E(\cdot, \cdot)$ be the fundamental solution of the heat equation and consider the single layer heat operator

$$
L_{\Gamma} u_{\Gamma}(x, t)=\int_{0}^{t} \int_{\Gamma} u_{\Gamma}(y, \tau) E(x-y, t-\tau) d s_{y} d \tau
$$

on the smooth closed curve $\Gamma$. Having the parametric representation $\theta \mapsto$ $x(\theta)$ of $\Gamma$ we put $u(\theta, t)=u_{\Gamma}(x(\theta), t)$ and define the operator

(b) $\left.\quad L u(\theta, t)=\int_{0}^{t} \int_{0}^{1} u(\phi, \tau) E(x(\theta)-x(\phi), t-\tau)\right)\left|x^{\prime}(\phi)\right| d \phi d \tau$.

If $\Gamma$ is a circle with radius $r$, then the operator $L$ has the principal symbol

$$
a(\xi, \eta)=\frac{r}{2\left(|\xi|^{2}+\mathrm{i} \eta r^{2}\right)^{\frac{1}{2}}} .
$$

The operator $L$ satisfies all the assumptions (2.1a-h), see [2, 8, 16, 24]. The formula (c) for the principal symbol can be determined by using [27], or more directly applying Laplace transform to the kernel representation (b) and utilizing the asymptotic formulae of [1] for the appearing Bessel functions. 
Example 2.2 Further examples of operators satisfying conditions (2.1) of arbitrarily high order can be obtained by defining

$A_{4 m+\beta} u(\theta, t)=\frac{1}{2 \pi} \sum_{n \neq 0} \int_{\mathbb{R}}\left(n^{4}+\eta^{2}\right)^{m}\left(\alpha n^{2}+\mathrm{i} \eta\right)^{\beta / 2} \hat{u}(n, \eta) \mathrm{e}^{\mathrm{i} n 2 \pi \theta+\mathrm{i} \eta t} d \eta$.

Here $m$ is a nonnegative integer, $-2<\beta<2, \alpha>0$. The order of $A_{4 m+\beta}$ is $4 m+\beta$.

Of particular importance are these operators with $m=0$. The principal symbol is in this case

$$
a_{\beta}(\xi, \eta)=\left(\alpha|\xi|^{2}+\mathrm{i} \eta\right)^{\beta / 2} .
$$

Note that the coercivity condition (2.1e) is satisfied for (2.3) if and only if $|\beta|<2$.

For shortness we write the representation (2.1b) as

$$
A u(x)=\frac{1}{2 \pi} \int_{\mathbb{Z} \times \mathbb{R}} a(\zeta) \hat{u}(\zeta) \mathrm{e}^{\mathrm{i}\langle x, \zeta\rangle} \underline{d \zeta},
$$

where $x=(\theta, t), \zeta=(n, \eta),\langle x, \zeta\rangle=n 2 \pi \theta+\eta t$, and the integration notation $\int d \zeta$ means the summation with respect of the first variable and integration with respect the second variable.

For our analysis the following modification of $A$ is useful. We introduce the modified symbol $a_{*}(\xi, \eta)$ putting

$$
a_{*}(\xi, \eta)= \begin{cases}\frac{1}{2}(1+\xi) a(1, \eta)+\frac{1}{2}(1-\xi) a(-1, \eta), & |\xi|<1, \eta \in \mathbb{R}, \\ a(\xi, \eta), & |\xi| \geq 1, \eta \in \mathbb{R}\end{cases}
$$

and define $A_{*}$ by the formula (2.1b) replacing $a$ with $a_{*}$. Now we have

$$
A_{*}: H^{s, \frac{s}{2}} \rightarrow H^{s-\beta, \frac{1}{2}(s-\beta)} \text { is an isomorphism, }
$$

$$
\begin{aligned}
& \operatorname{Re} a_{*}(\xi, \eta) \geq c_{0}\left(1+|\xi|+|\eta|^{\frac{1}{2}}\right)^{\beta}, \quad \xi, \eta \in \mathbb{R}, \\
& \operatorname{Re}\left(A_{*} u \mid u\right) \geq c_{0}^{\prime} \|\left. u\right|_{\alpha, \frac{\alpha}{2}} ^{2}, \quad u \in H^{\alpha, \frac{\alpha}{2}}, \alpha=\frac{\beta}{2},
\end{aligned}
$$

whereas $A$ need not to have these properties. Here $(u \mid v), u \in H^{s, \frac{s}{2}}, v \in$ $H^{-s,-\frac{s}{2}}$ denotes the natural continuation of the $L^{2}$-inner product.

\section{Collocation problem}

Let $N$ be a positive integer and $h_{t}>0$. We introduce the uniform meshes $\left\{\theta_{k}=k h_{\theta}\right\}, k \in \mathbb{Z}, h_{\theta}=\frac{1}{N}$ and $\left\{t_{l}=l h_{t}\right\}, l \in \mathbb{Z}$. If we are especially interested on the approximate solution on the interval $[0, T]$, it is natural 
to choose $h_{t}=\frac{T}{M}, M \in \mathbb{N}$. In the following analysis we apply spline collocation at the nodal points of the mesh. For this, let $S_{h_{\theta}}^{d_{\theta}}$ be the space of all 1-periodic smoothest splines of degree $d_{\theta} \in \mathbb{N}_{0}$ subordinate to the mesh $\left\{\theta_{k}\right\}$, if $d_{\theta}$ is an odd integer, and subordinate to the shifted mesh $\left\{\left(k+\frac{1}{2}\right) h_{\theta}\right\}$, if $d_{\theta}$ is an even integer. Correspondingly, let $V_{h_{t}}^{d_{t}}$ be the space of (non-periodic) splines of degree $d_{t}$ on $\mathbb{R}$. Observe that functions in $V_{h_{t}}^{d_{t}}$ have no growth condition at infinity. For our purpose it is more convenient to use the spaces $S_{h_{t}}^{d_{t}}=V_{h_{t}}^{d_{t}} \cap L^{2}(\mathbb{R})$. These spaces are infinite-dimensional and, assuming $N \geq d_{\theta}+1$, the spaces $S_{h_{\theta}}^{d_{\theta}}$ are $N$-dimensional. For approximation of functions $u(\theta, t)$ we use the tensor product spaces $S_{h_{\theta}}^{d_{\theta}} \times S_{h_{t}}^{d_{t}}$.

In this section we consider collocation approximation of the equation

$$
A_{*} u=f .
$$

The collocation problem is: find $u_{h} \in S_{h_{\theta}}^{d_{\theta}} \times S_{h_{t}}^{d_{t}}$ such that

$$
A_{*} u_{h}\left(x_{\kappa}\right)=f\left(x_{\kappa}\right), \quad \kappa \in \mathbb{Z}_{N} \times \mathbb{Z}
$$

where $x_{\kappa}=\left(\theta_{k}, t_{l}\right), \kappa=(k, l)$ and $\mathbb{Z}_{N}=\left\{n \in \mathbb{Z} \mid-\frac{N}{2}<n \leq \frac{N}{2}\right\}$. Observe that no initial condition is imposed in this whole space setting. We shall show that under certain assumptions the collocation equations are uniquely solvable. For the analysis we apply Fourier techniques. We recall the Fourier representation of spline functions. Let $\varpi$ be the characteristic function of the unit interval $\left(-\frac{1}{2}, \frac{1}{2}\right)$. We define the basic functions $\chi_{h_{t}, l}^{d_{t}}$ of $S_{h_{t}}^{d_{t}}$ by $\chi_{h_{t}, l}^{d_{t}}(\tau)=\varpi^{d_{t}}\left(h_{t}{ }^{-1} \tau-l\right), l \in \mathbb{Z}$, where $\varpi^{d_{t}}$ is the $d_{t}+1$-fold convolution of $\varpi$. Correspondingly, we have the 1-periodic basic splines $\phi_{h_{\theta}, k}^{d_{\theta}}, k \in \mathbb{Z}_{N}$ and the basis functions $\psi_{h, \kappa}^{d}=\phi_{h_{\theta}, k}^{d_{\theta}} \times \chi_{h_{t}, l}^{d_{t}}, \kappa \in \mathbb{Z}_{N} \times \mathbb{Z}$ for $S_{h_{\theta}}^{d_{\theta}} \times S_{h_{t}}^{d_{t}}$. Function $u_{h} \in S_{h_{\theta}}^{d_{\theta}} \times S_{h_{t}}^{d_{t}}$ has the unique representation $u_{h}=$ $u_{h}\left\{c_{\kappa}\right\}=\sum_{\kappa} c_{\kappa} \psi_{h, \kappa}^{d}$ such that $\left\{c_{\kappa}\right\} \in L^{2}\left(\mathbb{Z}_{N} \times \mathbb{Z}\right)$. Here $L^{2}\left(\mathbb{Z}_{N} \times \mathbb{Z}\right)$ is the space of the double sequences $\left\{c_{\kappa} \mid \kappa \in \mathbb{Z}_{N} \times \mathbb{Z}\right\}$ such that $\sum_{\kappa \in \mathbb{Z}_{N} \times \mathbb{Z}}\left|c_{\kappa}\right|^{2}<$ $\infty$. Using the Fourier transform $(\mathcal{F} \varpi)(\eta)=\frac{\sin (\eta / 2)}{\eta / 2}$ we obtain

$$
\begin{aligned}
\widehat{\psi_{h, \kappa}^{d}}(\zeta) & =\mathrm{e}^{-\mathrm{i}\left\langle x_{\kappa}, \zeta\right\rangle} \widehat{\psi_{h}^{d}}(\zeta) \\
& =h_{\theta} h_{t} \mathrm{e}^{-\mathrm{i}\left\langle x_{\kappa}, \zeta\right\rangle}\left[(\mathcal{F} \varpi)\left(2 \pi h_{\theta} n\right)\right]^{d_{\theta}+1}\left[(\mathcal{F} \varpi)\left(h_{t} \eta\right)\right]^{d_{t}+1},
\end{aligned}
$$

where $\psi_{h}^{d}=\psi_{h,(0,0)}^{d}$. By (3.3) we get the following recurrence relation for the Fourier coefficients of the spline functions $u_{h} \in S_{h_{\theta}}^{d_{\theta}} \times S_{h_{t}}^{d_{t}}$

$$
\widehat{u}_{h}\left(n+p N, \eta+q \frac{2 \pi}{h_{t}}\right)=(-1)^{\kappa_{p, q}}\left[\frac{n}{n+p N}\right]^{d_{\theta}+1}\left[\frac{\eta}{\eta+q \frac{2 \pi}{h_{t}}}\right]^{d_{t}+1} \widehat{u}_{h}(n, \eta),
$$


where $\kappa_{p, q}=p\left(d_{\theta}+1\right)+q\left(d_{t}+1\right)$. The essential tool in our analysis is the discrete Fourier transform together with a generalized version of the Poisson formula for functions in $H^{s, \frac{s}{2}}$. For the case of ordinary Sobolev spaces see [6]. We need the following Sobolev embedding result for anisotropic spaces [20]. We recall shortly also the proof of this basic result.

Theorem 3.1 Assume $s>\frac{3}{2}$. If $u \in H^{s, \frac{s}{2}}$, then $u$ is a continuous bounded function in $\mathbb{R}_{\theta} \times \mathbb{R}_{t}$ and the embedding $H^{s, \frac{s}{2}} \subset C\left(\mathbb{R}_{\theta} \times \mathbb{R}_{t}\right)$ is continuous such that

$$
\sup _{(\theta, t) \in \mathbb{R}_{\theta} \times \mathbb{R}_{t}}|u(\theta, t)| \leq c(s)\|u\|_{s, \frac{s}{2}}, \quad u \in H^{s, \frac{s}{2}}
$$

Proof. One easily verifies that the function

$$
I(s)=\sum_{n \in \mathbb{Z}} \int_{\mathbb{R}}\left(1+|n|+|\eta|^{\frac{1}{2}}\right)^{-2 s} d \eta, \quad s \in \mathbb{R}
$$

is finite if and only if $s>\frac{3}{2}$. Let $C_{10}^{\infty}$ be the space of the smooth functions $u(\theta, t)$ which are 1-periodic with respect to $\theta$ and vanish identically for sufficient large $t$. Since this space is dense in $H^{s, \frac{s}{2}}$ it is enough to prove (3.5) for $u \in C_{10}^{\infty}$. By using the Fourier representation of $u$ and the CauchySchwarz inequality, we obtain

$$
|u(\theta, t)| \leq \frac{1}{2 \pi} \sum_{n \in \mathbb{Z}} \int_{\mathbb{R}}|\hat{u}(n, \eta)| d \eta \leq \frac{\sqrt{I(s)}}{2 \pi}\|u\|_{s, \frac{s}{2}} . \square
$$

The classical Poisson formula extends for functions $u \in H^{s, \frac{s}{2}}, s>\frac{3}{2}$. In this connection we introduce the discrete Fourier transform for sequences and functions. Having a sequence $\underline{c}=\left\{c_{\kappa}\right\} \in L^{2}\left(\mathbb{Z}_{N} \times \mathbb{Z}\right)$ the discrete Fourier transform $\underline{\tilde{c}}(\zeta), \zeta \in \mathbb{Z}_{N} \times \mathbb{R}_{h_{t}}, \mathbb{R}_{h_{t}}=\left(-\frac{\pi}{h_{t}}, \frac{\pi}{h_{t}}\right)$ of $\underline{c}$ is defined as

$$
\tilde{\tilde{c}}(\zeta)=h_{\theta} h_{t} \sum_{\kappa \in \mathbb{Z}_{N} \times \mathbb{Z}} c_{\kappa} \mathrm{e}^{-\mathrm{i}\left\langle x_{\kappa}, \zeta\right\rangle}, \quad \zeta \in \mathbb{Z}_{N} \times \mathbb{R}_{h_{t}} .
$$

We have $\underline{\tilde{c}} \in L^{2}\left(\mathbb{Z}_{N} \times \mathbb{R}_{h_{t}}\right)$ and the mapping $\underline{c} \mapsto \underline{\tilde{c}}$ from $L^{2}\left(\mathbb{Z}_{N} \times \mathbb{Z}\right)$ to $L^{2}\left(\mathbb{Z}_{N} \times \mathbb{R}_{h_{t}}\right)$ is an isomorphism such that

$$
(\underline{c} \mid \underline{d})_{h}=\frac{1}{2 \pi}(\underline{\tilde{c}} \mid \underline{\tilde{d}})_{L^{2}\left(\mathbb{Z}_{N} \times \mathbb{R}_{h_{t}}\right)}
$$

where the inner-products are defined by

$$
\begin{aligned}
& (\underline{c} \mid \underline{d})_{h}=h_{\theta} h_{t} \sum_{\kappa \in \mathbb{Z}_{N} \times \mathbb{Z}} c_{\kappa} \bar{d}_{\kappa}, \\
& (\underline{\tilde{c}} \mid \underline{\tilde{d}})_{L^{2}\left(\mathbb{Z}_{N} \times \mathbb{R}_{h_{t}}\right)}=\int_{\mathbb{Z}_{N} \times \mathbb{R}_{h_{t}}} \tilde{\underline{c}}(\zeta) \overline{\tilde{d}}(\zeta) \underline{d} \zeta .
\end{aligned}
$$


The inverse mapping $\underline{\tilde{c}} \mapsto \underline{c}$ is given by

$$
c_{\kappa}=\frac{1}{2 \pi} \int_{\mathbb{Z}_{N} \times \mathbb{R}_{h_{t}}} \underline{\tilde{c}}(\zeta) \mathrm{e}^{\mathrm{i}\left\langle x_{\kappa}, \zeta\right\rangle} \underline{d} \zeta, \kappa \in \mathbb{Z}_{N} \times \mathbb{Z} .
$$

If the point values $\left\{u\left(x_{\kappa}\right)\right\}$ of the function $u$ are given and $\left\{u\left(x_{\kappa}\right)\right\} \in$ $L^{2}\left(\mathbb{Z}_{N} \times \mathbb{Z}\right)$, then the discrete Fourier transform of $u$ is defined by

$$
\tilde{u}(\zeta)=h_{\theta} h_{t} \sum_{\kappa \in \mathbb{Z}_{N} \times \mathbb{Z}} u\left(x_{\kappa}\right) \mathrm{e}^{-\mathrm{i}\left\langle x_{\kappa}, \zeta\right\rangle}, \quad \zeta \in \mathbb{Z}_{N} \times \mathbb{R}_{h_{t}} .
$$

The next two theorems are very essential for our work. Applying these theorems we get an effective formulation for collocation equations on uniform meshes.

Theorem 3.2 (Poisson formula). Assume that $u \in H^{s, \frac{s}{2}}, s>\frac{3}{2}$ and $h_{0 t}>0$. Then $\left\{u\left(x_{\kappa}\right)\right\} \in L^{2}\left(\mathbb{Z}_{N} \times \mathbb{Z}\right)$ and we have

(3.11a) $\tilde{u}(\zeta)=\sum_{p, q \in \mathbb{Z}} \hat{u}\left(n+p N, \eta+q \frac{2 \pi}{h_{t}}\right)$, in $L^{2}\left(\mathbb{Z}_{N} \times \mathbb{R}_{h_{t}}\right)$,

(3.11b) $2 \pi h_{\theta} h_{t} \sum_{\kappa \in \mathbb{Z}_{N} \times \mathbb{Z}}\left|u\left(x_{\kappa}\right)\right|^{2}=\|\tilde{u}\|_{L^{2}\left(\mathbb{Z}_{N} \times \mathbb{R}_{h_{t}}\right)}^{2} \leq c\left(s, h_{0 t}\right)\|u\|_{s, \frac{s}{2}}^{2}$,

where (3.11b) holds for $0<h_{t} \leq h_{0 t}$.

Proof. Suppose that $u \in C_{10}^{\infty}$. By applying the discrete Fourier transform for 1-periodic functions and the Poisson summation formula for non-periodic functions we have

$$
\begin{aligned}
\frac{1}{N} \sum_{k \in \mathbb{Z}_{N}} u\left(\theta_{k}, t\right) \mathrm{e}^{-\mathrm{i} n 2 \pi \theta_{k}} & =\sum_{p \in \mathbb{Z}} \hat{u}(n+p N, t),(\hat{u}(\cdot, t) \text { is F-coefficient }) \\
h_{t} \sum_{l \in \mathbb{Z}} u\left(\theta, t_{l}\right) \mathrm{e}^{-\mathrm{i} \eta t_{l}} & =\sum_{q \in \mathbb{Z}} \hat{u}\left(\theta, \eta+q \frac{2 \pi}{h_{t}}\right),(\hat{u}(\theta, \cdot) \text { is F-transform })
\end{aligned}
$$

which imply (3.11a) for functions in $C_{10}^{\infty}$. By (3.7) we have

$$
2 \pi h_{\theta} h_{t} \sum_{\kappa \in \mathbb{Z}_{N} \times \mathbb{Z}}\left|u\left(x_{\kappa}\right)\right|^{2}=\sum_{n \in \mathbb{Z}_{N}} \int_{-\pi / h_{t}}^{\pi / h_{t}}\left|\sum_{p, q} \hat{u}\left(n+p N, \eta+q \frac{2 \pi}{h_{t}}\right)\right|^{2} d \eta=: T .
$$

We will show that (3.12) extends to $H^{s, \frac{s}{2}}, s>\frac{3}{2}$ by continuity. To this end we estimate the right-hand side of (3.12) for functions $u \in H^{s, \frac{s}{2}}$. 
For $s>\frac{3}{2}, h_{0 t}>0$ there is a positive number $c\left(s, h_{0 t}\right)$ such that for all $(n, \eta) \in \mathbb{Z}_{N} \times \mathbb{R}_{h_{t}}, 0<h_{t} \leq h_{0 t}$ holds

$$
\sum_{(p, q) \neq(0,0)}\left(|n+p N|+\left|\eta+q \frac{2 \pi}{h_{t}}\right|^{\frac{1}{2}}\right)^{-2 s} \leq c\left(s, h_{0 t}\right) .
$$

By this estimate we obtain $T \leq\|u\|_{0,0}^{2}+c\left(s, h_{0 t}\right) T_{1}$ where

$$
\begin{aligned}
T_{1} & =\sum_{n \in \mathbb{Z}_{N}} \int_{\mathbb{R}_{h_{t}}} \sum_{(p, q) \neq(0,0)}\left(|n+p N|+\left|\eta+q \frac{2 \pi}{h_{t}}\right|^{\frac{1}{2}}\right)^{2 s}\left|\hat{u}\left(n+p N, \eta+q \frac{2 \pi}{h_{t}}\right)\right|^{2} d \eta \\
& \leq c\|u\|_{s, \frac{s}{2}}^{2} .
\end{aligned}
$$

Thus we have proved for $u \in H^{s, \frac{s}{2}}, 0<h_{t} \leq h_{0 t}, s>\frac{3}{2}$

$$
\sum_{n \in \mathbb{Z}_{N}} \int_{-\pi / h_{t}}^{\pi / h_{t}}\left|\sum_{p, q} \hat{u}\left(n+p N, \eta+q \frac{2 \pi}{h_{t}}\right)\right|^{2} d \eta \leq c\left(s, h_{0 t}\right)\|u\|_{s, \frac{s}{2}}^{2} .
$$

Next we show that $\left\{u\left(x_{\kappa}\right)\right\} \in L^{2}\left(\mathbb{Z}_{N} \times \mathbb{Z}\right)$ for $u \in H^{s, \frac{s}{2}}, s>\frac{3}{2}$. Take a sequence $\left\{u_{n}\right\} \in C_{10}^{\infty}$ such that $\left\{u_{n}\right\}$ converges to $u$ in $H^{s, \frac{s}{2}}$. Applying (3.12), (3.13) to $u_{n}-u_{m}$ we find that $\left\{u_{n}\left(x_{\kappa}\right)\right\}$ is a Cauchy sequence in $L^{2}\left(\mathbb{Z}_{N} \times \mathbb{Z}\right)$ and has a limit $\left\{u_{\kappa}\right\} \in L^{2}\left(\mathbb{Z}_{N} \times \mathbb{Z}\right)$. By the Sobolev embedding follows $u\left(x_{\kappa}\right)=u_{\kappa}$, hence $\left\{u\left(x_{\kappa}\right)\right\} \in L^{2}\left(\mathbb{Z}_{N} \times \mathbb{Z}\right)$. By a limiting process we finally obtain that (3.11a), (3.11b) are valid for $u \in H^{s, \frac{s}{2}}, s>\frac{3}{2}$.

Theorem 3.2 does not give accurate results when applied to functions $A_{*} u_{h}, u_{h} \in S_{h_{\theta}}^{d_{\theta}} \times S_{h_{t}}^{d_{t}}$. There holds $S_{h_{\theta}}^{d_{\theta}} \times S_{h_{t}}^{d_{t}} \subset H^{s, \frac{s}{2}}, s<\min \left\{d_{\theta}+\right.$ $\left.\frac{1}{2}, 2 d_{t}+1\right\}$, and Theorem 3.2 implies that $A_{*} u_{h}$ is continuous and the discrete Fourier transform $\widetilde{A_{*} u_{h}}$ is defined if $\beta<\min \left\{d_{\theta}-1,2 d_{t}-\frac{1}{2}\right\}$. We can replace this condition with the following

$$
\beta<\min \left\{d_{\theta}, 2 d_{t}\right\} .
$$

In addition, we assume that one of the following conditions is satisfied

(3.14b) Integers $d_{\theta}, d_{t}$ are odd,

(3.14c) $d_{\theta}+d_{t}$ is odd, $|\beta|<2, \beta \geq-d_{\theta}-1$, the principal symbol is as in (2.3),

(3.14d) Integers $d_{\theta}, d_{t}$ are even, (2.3) holds with $\beta=-1$.

We define $\mathbb{N}_{*}^{2}=\left\{(p, q) \in \mathbb{N}_{0}^{2} \mid(p, q) \neq(0,0)\right\}$. Assuming $\lambda \geq 1, \mu \geq 1, \nu<$ $\min \{\lambda-1,2(\mu-1)\}$, one easily verifies

$$
\sum_{(p, q) \in \mathbb{N}_{*}^{2}}\left(p+q^{\frac{1}{2}}\right)^{\nu}(1+p)^{-\lambda}(1+q)^{-\mu}<\infty .
$$


Theorem 3.3 Assume (3.14) and let $u_{h}\left\{c_{\kappa}\right\} \in S_{h_{\theta}}^{d_{\theta}} \times S_{h_{t}}^{d_{t}}$. Then $A_{*} u_{h}$ is continuous, the discrete Fourier transform $\widetilde{A_{*} u_{h}}$ is defined and we have

$$
\widetilde{A_{*} u_{h}}=\frac{1}{h_{\theta} h_{t}} \widetilde{\tilde{c}} \widetilde{A_{*} \psi_{h}^{d}} .
$$

Proof. From (3.3) we deduce

$$
\hat{u}_{h}(\zeta)=\frac{1}{h_{\theta} h_{t}} \tilde{\tilde{c}}(\zeta) \widehat{\psi_{h}^{d}}(\zeta), \quad \zeta \in \mathbb{Z} \times \mathbb{R} .
$$

Observe that $\underline{\tilde{c}}$ is a $\left(N, \frac{2 \pi}{h_{t}}\right)$-periodic function and $\hat{u}_{h} \in L^{2}(\mathbb{Z} \times \mathbb{R})$. Introducing $\zeta_{p, q}=\zeta+\left(p N, q \frac{2 \pi}{h_{t}}\right), p, q \in \mathbb{Z}$ we have

$$
A_{*} u_{h}(x)=\frac{1}{2 \pi h_{\theta} h_{t}} \sum_{p, q} \int_{\mathbb{Z}_{N} \times \mathbb{R}_{h_{t}}} a_{*}\left(\zeta_{p, q}\right) \widehat{\psi_{h}^{d}}\left(\zeta_{p, q}\right) \tilde{\tilde{c}}(\zeta) \mathrm{e}^{\mathrm{i}\left\langle x, \zeta_{p, q}\right\rangle} \underline{d}
$$

For fixed $N, h_{t}$ there holds

$$
\left|a_{*}\left(\zeta_{p, q}\right) \widehat{\psi_{h}^{d}}\left(\zeta_{p, q}\right)\right| \leq c\left(1+|p|+|q|^{\frac{1}{2}}\right)^{\beta}(1+|p|)^{-d_{\theta}-1}(1+|q|)^{-d_{t}-1}
$$

for $\zeta \in \mathbb{Z}_{N} \times \mathbb{R}_{h_{t}}$. The continuity of $A_{*} u_{h}(x)$ follows by using (3.15), the continuity and uniform boundedness of the functions $\mathrm{e}^{\mathrm{i}\left\langle x, \zeta_{p, q}\right\rangle}$ and the fact that the $L^{2}\left(\mathbb{Z}_{N} \times \mathbb{R}_{h_{t}}\right)$-function $\underline{\tilde{\tilde{c}}}(\zeta)$ is absolutely integrable over $\mathbb{Z}_{N} \times \mathbb{R}_{h_{t}}$ . Introducing the temporary notation

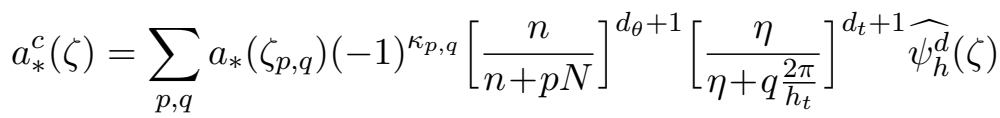

we have for the collocation points $x=x_{\kappa}$

$$
A_{*} u_{h}\left(x_{\kappa}\right)=\frac{1}{2 \pi h_{\theta} h_{t}} \int_{\mathbb{Z}_{N} \times \mathbb{R}_{h_{t}}} a_{*}^{c}(\zeta) \tilde{\tilde{c}}(\zeta) \mathrm{e}^{\mathrm{i}\left\langle x_{\kappa}, \zeta\right\rangle} \underline{d} \zeta .
$$

By this formula the values $A_{*} u_{h}\left(x_{\kappa}\right)$ are the Fourier coefficients of the $L^{2}\left(\mathbb{Z}_{N} \times \mathbb{R}_{h_{t}}\right)$-function $\frac{1}{2 \pi h_{\theta} h_{t}} a_{*}^{c} \underline{\tilde{c}}$. Therefore $\left\{A_{*} u_{h}\left(x_{\kappa}\right)\right\} \in L^{2}\left(\mathbb{Z}_{N} \times \mathbb{Z}\right)$ and $\widetilde{A_{*} u_{h}}$ is well-defined. But using the definition (3.10) for $\widetilde{A_{*} u_{h}}$ and (3.9) we obtain

$$
A_{*} u_{h}\left(x_{\kappa}\right)=\frac{1}{2 \pi} \int_{\mathbb{Z}_{N} \times \mathbb{R}_{h_{t}}} \widetilde{A_{*} u_{h}}(\zeta) \mathrm{e}^{\mathrm{i}\left\langle x_{\kappa}, \zeta\right\rangle} \underline{d \zeta} .
$$

Since $\left\{\mathrm{e}^{\mathrm{i}\left\langle x_{\kappa}, \zeta\right\rangle}\right\}$ is a complete basis of $L^{2}\left(\mathbb{Z}_{N} \times \mathbb{R}_{h_{t}}\right)$, (3.20), (3.21) imply $\widetilde{A_{*} u_{h}}=\frac{1}{h_{\theta} h_{t}} a_{*}^{c} \tilde{\tilde{c}}$. Finally, choosing $u_{h}=\psi_{h}^{d}$ we get $\widetilde{A_{*} \psi_{h}^{d}}=a_{*}^{c}$ which yields (3.16). 
To obtain solvability of the problem (3.2) we analyze the function $\widetilde{A_{*} \psi_{h}^{d}}$ which can be considered as an approximation of $\widehat{A_{*} \psi_{h}^{d}}$. Henceforth $c, c_{0}$ denote general positive constants which are independent of the discretization parameters. Observe that $c_{0} h_{\theta} h_{t} \leq \widehat{\psi_{h}^{d}}(\zeta) \leq c h_{\theta} h_{t}, \zeta \in \mathbb{Z}_{N} \times \mathbb{R}_{h_{t}}$. Furthermore, there holds for all $\zeta \in \mathbb{Z}_{N} \times \mathbb{R}_{h_{t}}$

$$
\begin{gathered}
c_{0} h_{\theta} h_{t}|\underline{\zeta}|^{\beta} \leq\left|\widehat{A_{*} \psi_{h}^{d}}(\zeta)\right| \leq c h_{\theta} h_{t} \mid \underline{\zeta}^{\beta}, \\
\operatorname{Re} \widehat{A_{*} \psi_{h}^{d}}(\zeta) \geq c_{0} h_{\theta} h_{t}|\underline{\zeta}|^{\beta},
\end{gathered}
$$

where $|\underline{\zeta}|=|\underline{n}|+|\eta|^{1 / 2}$ with $|\underline{n}|=\max \{1,|n|\}$. Under some additional assumptions we show that the properties (3.22) remain valid when $\widehat{A_{*} \psi_{h}^{d}}$ is replaced by $\widetilde{A_{*} \psi_{h}^{d}}$. Moreover, we estimate the approximation error. We impose the condition

$$
\gamma^{2} / \pi \leq \nu:=h_{\theta}^{2} h_{t}^{-1} \leq \nu_{1}<\infty,
$$

where $\gamma$ is the parameter appearing in (2.2). As it was pointed out in Remark 2.1 the constant $\gamma$ can be taken arbitrary small for the classical parabolic boundary integral operators. Having the condition (3.23) we can use the common parameter $h=h_{\theta}$ and have then $h_{t} \sim h^{2}$. This condition is wellknown in connection of the parabolic problems.

Lemma 3.4 Assume (3.14) and (3.23). Abbreviating $\bar{d}=\min \left\{d_{\theta}, 2 d_{t}+1\right\}$ we have for all $\zeta \in \mathbb{Z}_{N} \times \mathbb{R}_{h_{t}}$,

$$
\begin{gathered}
c_{0} h^{3}|\underline{\zeta}|^{\beta} \leq\left|\widetilde{A_{*} \psi_{h}^{d}}(\zeta)\right| \leq c h^{3}|\underline{\zeta}|^{\beta}, \\
\operatorname{Re} \widetilde{A_{*} \psi_{h}^{d}}(\zeta) \geq c_{0} h^{3}|\underline{\zeta}|^{\beta}, \\
\left|\widetilde{A_{*} \psi_{h}^{d}}(\zeta)-\widetilde{A_{*} \psi_{h}^{d}}(\zeta)\right| \leq c[h|\underline{\zeta}|]^{\bar{d}+1-\beta}\left|\widetilde{A_{*} \psi_{h}^{d}}(\zeta)\right| .
\end{gathered}
$$

Proof. We have

$$
\begin{aligned}
\widetilde{A_{*} \psi_{h}^{d}}(\zeta) & =\widehat{A_{*} \psi_{h}^{d}}(\zeta) \\
& +\sum_{(p, q) \neq(0,0)} a_{*}\left(\zeta_{p, q}\right)(-1)^{\kappa_{p, q}}\left[\frac{n}{n+p N}\right]^{d_{\theta}+1}\left[\frac{\eta}{\eta+q \frac{2 \pi}{h_{t}}}\right]^{d_{t}+1} \widehat{\psi_{h}^{d}}(\zeta) .
\end{aligned}
$$

We assume that (3.14b) is valid. The proof for the cases (3.14c) and (3.14d) is given in the appendix. If both $d_{\theta}$ and $d_{t}$ are odd, then $(-1)^{\kappa_{p, q}}=+1$, and we obtain the stability estimate (or "ellipticity of the numerical symbol") (3.24b) by (2.1e),

$$
\operatorname{Re} \widetilde{A_{*} \psi_{h}^{d}}(\zeta) \geq \operatorname{Re} \widehat{A_{*} \psi_{h}^{d}}(\zeta) \geq c_{0} h_{\theta} h_{t}|\underline{\zeta}|^{\beta} .
$$


In order to prove the consistency estimate (3.24c), we consider the discretization error writing

$$
\widetilde{A_{*} \psi_{h}^{d}}(\zeta)-\widehat{A_{*} \psi_{h}^{d}}(\zeta)=T(\zeta) \widehat{\psi_{h}^{d}}(\zeta),
$$

where $T=T_{1}+T_{2}+T_{3}$ with

$$
\begin{aligned}
T_{1} & =\sum_{p \neq 0} a_{*}\left(\zeta_{p, 0}\right)(-1)^{\kappa_{p, 0}}\left[\frac{n}{n+p N}\right]^{d_{\theta}+1}, \\
T_{2} & =\sum_{q \neq 0} a_{*}\left(\zeta_{0, q}\right)(-1)^{\kappa_{0, q}}\left[\frac{\eta}{\eta+q \frac{2 \pi}{h_{t}}}\right]^{d_{t}+1}, \\
T_{3} & =\sum_{p, q \neq 0} a_{*}\left(\zeta_{p, q}\right)(-1)^{\kappa_{p, q}}\left[\frac{n}{n+p N}\right]^{d_{\theta}+1}\left[\frac{\eta}{\eta+q \frac{2 \pi}{h_{t}}}\right]^{d_{t}+1} .
\end{aligned}
$$

Assume $n \neq 0$. Using (2.1d), (2.2) we obtain for all $(p, q) \neq(0,0)$

$$
\begin{aligned}
\left|a_{*}\left(\zeta_{p, q}\right)\right| & =\left|a\left(\zeta_{p, q}\right)\right|=N^{\beta}\left|a\left(p+\frac{n}{N}, \nu\left(q 2 \pi+h_{t} \eta\right)\right)\right| \\
& \leq c N^{\beta}\left(\left|p+\frac{n}{N}\right|+\left|\nu\left(q 2 \pi+h_{t} \eta\right)\right|^{\frac{1}{2}}\right)^{\beta} \leq c N^{\beta}\left(|p|+|q|^{\frac{1}{2}}\right)^{\beta},
\end{aligned}
$$

since $\left|p+\frac{n}{N}\right|+\left|\nu\left(q 2 \pi+h_{t} \eta\right)\right|^{\frac{1}{2}} \geq \gamma$ if $\nu \geq \gamma^{2} / \pi$. Therefore by (3.15), $d_{\theta}>\beta, 2 d_{t}>\beta$

$$
\begin{aligned}
\left|T_{3}\right| & \leq c N^{\beta}\left|\frac{n}{N}\right|^{d_{\theta}+1}\left|h_{t} \eta\right|^{d_{t}+1} \sum_{p, q=1}^{\infty}\left(p+q^{\frac{1}{2}}\right)^{\beta} \frac{1}{p^{d_{\theta}+1}} \frac{1}{q^{d_{t}+1}} \\
& \leq c h^{-\beta}|h \underline{n}|^{d_{\theta}+1}\left|h^{2} \eta\right|^{d_{t}+1} .
\end{aligned}
$$

The other terms have the bounds $\left|T_{1}\right| \leq c h^{-\beta}|h \underline{n}|^{d_{\theta}+1},\left|T_{2}\right| \leq c h^{-\beta}$ $\cdot\left|h^{2} \eta\right|^{d_{t}+1}$. These upper bounds remain valid also for $n=0$ and we obtain $|T| \leq c h^{-\beta}[h|\underline{\zeta}|]^{\bar{d}+1}$ which yields

$$
\left|\widetilde{A_{*} \psi_{h}^{d}}(\zeta)-\widehat{A_{*} \psi_{h}^{d}}(\zeta)\right| \leq c[h|\underline{\zeta}|]^{\bar{d}+1-\beta}\left|\widehat{A_{*} \psi_{h}^{d}}(\zeta)\right| .
$$

Since $\bar{d}+1-\beta \geq 1$ and $h|\underline{\zeta}|$ is uniformly bounded in $\mathbb{Z}_{N} \times \mathbb{R}_{h_{t}}$, (3.26) implies

$$
\left|\widetilde{A_{*} \psi_{h}^{d}}(\zeta)\right| \leq c\left|\widehat{A_{*} \psi_{h}^{d}}(\zeta)\right| .
$$

Using (3.27), (3.24b), (3.22a) we get (3.24a) and (3.24c).

We assume that for the function $f$ there holds $f=\left\{f\left(x_{\kappa}\right)\right\} \in L^{2}\left(\mathbb{Z}_{N} \times\right.$ $\mathbb{Z}$ ). Then using the representation $u_{h}=u_{h}\left\{c_{\kappa}\right\}$ for $u_{h} \in S_{h_{\theta}}^{d_{\theta}} \times S_{h_{t}}^{d_{t}}$, we can give the collocation problem in the form

$$
\mathcal{A}_{*} \underline{c}=\underline{f},
$$


where $\mathcal{A}_{*}$ is defined by $\mathcal{A}_{*} \underline{c}=\left\{\left(A_{*} u_{h}\right)\left(x_{\kappa}\right)\right\}$. Observe that $\mathcal{A}_{*}$ depends on the parameters $d, N$ and $h_{t}$. It turns out that $\mathcal{A}_{*}: L^{2}\left(\mathbb{Z}_{N} \times \mathbb{Z}\right) \rightarrow$ $L^{2}\left(\mathbb{Z}_{N} \times \mathbb{Z}\right)$ is an isomorphism. In particular, the collocation problem is uniquely solvable. To prove that result, we introduce in $L^{2}\left(\mathbb{Z}_{N} \times \mathbb{Z}\right)$ the discrete norms $\|\underline{c}\|_{s, \frac{s}{2} ; h}, s \in \mathbb{R}$ such that

$$
\|\left.\underline{c}\right|_{s, \frac{s}{2} ; h} ^{2}=\frac{1}{2 \pi} \int_{\mathbb{Z}_{N} \times \mathbb{R}_{h_{t}}}|\underline{\zeta}|^{2 s}|\underline{\tilde{c}}(\zeta)|^{2} \underline{d} \zeta .
$$

The discrete anisotropic Sobolev space $H_{h}^{s, \frac{s}{2}}$ is the space $L^{2}\left(\mathbb{Z}_{N} \times \mathbb{Z}\right)$ endowed with norm $\|\underline{c}\|_{s, \frac{s}{2} ; h}$. Observe that for fixed parameters $N$ and $h_{t}$ all the norms $\|\underline{c}\|_{s, \frac{s}{2} ; h}, s \in \mathbb{R}$, are equivalent. With $s=0$ we have $\|\underline{c}\|_{s, \frac{s}{2} ; h}=\|\underline{c}\|_{h}$. Moreover we have

$$
\left|(\underline{c} \mid \underline{d})_{h}\right| \leq\left\|\left.\underline{c}\right|_{s, \frac{s}{2} ; h}|| \underline{d}\right\|_{-s,-\frac{s}{2} ; h}, \quad s \in \mathbb{R} .
$$

Using these norms we can describe the mapping properties of $\mathcal{A}_{*}$ very much analogously with the continuous case. In addition to the whole space problem (3.2) we consider the corresponding problem where the vanishing initial condition is taken into account. In the case of this latter problem we assume that $d_{t}=0$ or $d_{t}=1$. We define

$$
\tilde{S}_{h_{t}}^{d_{t}}=\left\{v \in S_{h_{t}}^{d_{t}} \mid \operatorname{supp} v \subset[0, \infty)\right\} .
$$

Under our assumption concerning the degree $d_{t}$ there holds

$$
S_{h_{\theta}}^{d_{\theta}} \times \tilde{S}_{h_{t}}^{d_{t}}=\left\{u_{h}=u_{h}\left\{c_{\kappa}\right\} \in S_{h_{\theta}}^{d_{\theta}} \times S_{h_{t}}^{d_{t}} \mid \underline{c} \in \tilde{L}^{2}\left(\mathbb{Z}_{N} \times \mathbb{Z}\right)\right\},
$$

where $\tilde{L}^{2}\left(\mathbb{Z}_{N} \times \mathbb{Z}\right)=\left\{\underline{c} \in L^{2}\left(\mathbb{Z}_{N} \times \mathbb{Z}\right) \mid c_{k, l}=0, k, l \in \mathbb{Z}, l \leq 0\right\}$. We set

$$
\tilde{H}_{h}^{s, \frac{s}{2}}=\left\{\underline{c} \in H_{h}^{s, \frac{s}{2}} \mid \underline{c} \in \tilde{L}^{2}\left(\mathbb{Z}_{N} \times \mathbb{Z}\right)\right\} .
$$

The half space problem with the vanishing initial condition is given by: for $f=A_{*} u, u \in \tilde{H}^{s, \frac{s}{2}}$ find $u_{h} \in S_{h_{\theta}}^{d_{\theta}} \times \tilde{S}_{h_{t}}^{d_{t}}$ such that

$$
A_{*} u_{h}\left(x_{\kappa}\right)=f\left(x_{\kappa}\right), \quad \kappa \in \mathbb{Z}_{N} \times \mathbb{N} \text {. }
$$

Theorem 3.5 Assume (3.14) and (3.23). If $u \in H^{s, \frac{s}{2}}, s>\beta+\frac{3}{2}$, then the collocation problem (3.2) has a unique solution. If, in addition, $u \in \tilde{H}^{s, \frac{s}{2}}$ and $d_{t}=0$ or $d_{t}=1$, then the problem (3.30) is uniquely solvable and the solutions of (3.2) and (3.30) coincide. 
Proof. Consider the problem (3.2). We show that the operator $\mathcal{A}_{*}: H_{h}^{s, \frac{s}{2}} \rightarrow$ $H_{h}^{s-\beta, \frac{1}{2}(s-\beta)}$ is an isomorphism. First we prove the continuity. We have

$$
\widetilde{\mathcal{A}_{*} \underline{c}}=\widetilde{A_{*} u_{h}}=\frac{1}{h_{\theta} h_{t}} \tilde{\tilde{c}} \widetilde{A_{*} \psi_{h}^{d}} .
$$

By (3.24a), (3.31) we get the required continuity,

$$
\begin{aligned}
\|\left.\mathcal{A}_{*} \underline{c}\right|_{s-\beta, \frac{1}{2}(s-\beta) ; h} ^{2} & \leq c \int_{\mathbb{Z}_{N} \times \mathbb{R}_{h_{t}}}|\underline{\zeta}|^{2(s-\beta)}\left|\widetilde{A_{*} u_{h}}(\zeta)\right|^{2} \underline{d} \zeta \\
& \leq c \int_{\mathbb{Z}_{N} \times \mathbb{R}_{h_{t}}}|\underline{\zeta}|^{2 s}|\underline{\tilde{c}}(\zeta)|^{2} \underline{d} \zeta \leq c \|\left.\underline{c}\right|_{s, \frac{s}{2} ; h} ^{2} .
\end{aligned}
$$

Now, the equation (3.28) is equivalent with the problem: find $\underline{c} \in H_{h}^{\alpha, \frac{\alpha}{2}}$ such that

$$
\left(\mathcal{A}_{*} \underline{c} \mid \underline{d}\right)_{h}=(\underline{f} \mid \underline{d})_{h}, \underline{d} \in H_{h}^{\alpha, \frac{\alpha}{2}} .
$$

Since $\mathcal{A}_{*}: H_{h}^{\alpha, \frac{\alpha}{2}} \rightarrow H_{h}^{-\alpha,-\frac{\alpha}{2}}$ is continuous, the left-hand side of (3.32) defines a bounded sesquilinear form in $H_{h}^{\alpha, \frac{\alpha}{2}} \times H_{h}^{\alpha, \frac{\alpha}{2}}$. Moreover, by (3.7), (3.31),

$$
\left(\mathcal{A}_{*} \mid \underline{c}\right)_{h}=\frac{1}{2 \pi}\left(\widetilde{\mathcal{A}_{*} \underline{c}} \mid \underline{\tilde{c}}\right)_{\mathbb{Z}_{N} \times \mathbb{R}_{h_{t}}}=\frac{1}{2 \pi h_{\theta} h_{t}} \int_{\mathbb{Z}_{N} \times \mathbb{R}_{h_{t}}} \widetilde{A_{*} \psi_{h}^{d}}(\zeta)|\tilde{c}(\zeta)|^{2} \underline{d} \zeta .
$$

Using (3.24b) we get

$$
\operatorname{Re}\left(\mathcal{A}_{* \underline{c}} \mid \underline{c}\right)_{h} \geq c_{0} \int_{\mathbb{Z}_{N} \times \mathbb{R}_{h_{t}}}|\underline{\zeta}|^{\beta}|\underline{\tilde{c}}(\zeta)|^{2} \underline{d} \zeta \geq c_{0} \|\left.\underline{c}\right|_{\alpha, \frac{\alpha}{2} ; h} ^{2} .
$$

The unique solvability of (3.32) follows from the theorem of Lax and Milgram. Moreover, $\mathcal{A}_{*}: H_{h}^{\alpha, \frac{\alpha}{2}} \rightarrow H_{h}^{-\alpha,-\frac{\alpha}{2}}$ is an isomorphism such that $\left\|\mathcal{A}_{*}^{-1} \underline{f}\right\|_{\alpha, \frac{\alpha}{2} ; h}=\left\|\left.\underline{c}\right|_{\alpha, \frac{\alpha}{2} ; h} \leq c_{0}^{-1}|| \underline{f}\right\|_{-\alpha,-\frac{\alpha}{2} ; h}$. This proves the assertion for the case of (3.2). If $u \in \tilde{H}^{s, \frac{s}{2}}$ we have by the Volterra property that $f \in \tilde{H}_{h}^{-\alpha,-\frac{\alpha}{2}}$. The problem (3.30) is equivalent with the equation (3.28) such that $\underline{c} \in \tilde{H}_{h}^{\alpha, \frac{\alpha}{2}}$. By the Volterra property of $A_{*}$ and the continuity of $A_{*} u_{h}$ it follows that $\mathcal{A}_{*}$ maps $\tilde{H}_{h}^{s, \frac{s}{2}}$ into the space $\tilde{H}_{h}^{s-\beta, \frac{1}{2}(s-\beta)}$. Using the formulation (3.32) in the subspace $\tilde{H}_{h}^{-\alpha,-\frac{\alpha}{2}}$ we obtain the unique solution as in the previous case. But this solution satisfies also the equation (3.32) and therefore coincides with the whole-space solution. 
Remark 3.1 In fact we have an explicit formula for the Fourier coefficients of the collocation solution and it will be used in the next section. By Theorem 3.2 and Theorem 3.3 the collocation problem is equivalent with the equation

$$
\widetilde{A_{*} u_{h}}=\tilde{f}, \quad \text { in } \mathbb{Z}_{N} \times \mathbb{R}_{h_{t}} .
$$

By (3.16) this becomes

$$
\frac{1}{h_{\theta} h_{t}} \tilde{\tilde{c}} \widetilde{A_{*} \psi_{h}^{d}}=\tilde{f}
$$

By Lemma 3.4 the function $\left(\widetilde{A_{*} \psi_{h}^{d}}\right)^{-1}$ is well-defined and $\left(\widetilde{A_{*} \psi_{h}^{d}}\right)^{-1} \in$ $L^{\infty}\left(\mathbb{Z}_{N} \times \mathbb{R}_{h_{t}}\right)$. Since $\tilde{f} \in L^{2}\left(\mathbb{Z}_{N} \times \mathbb{R}_{h_{t}}\right)$ equation (3.35) has unique solution $\underline{\tilde{c}} \in L^{2}\left(\mathbb{Z}_{N} \times \mathbb{R}_{h_{t}}\right)$,

$$
\underline{\tilde{c}}=h_{\theta} h_{t}\left(\widetilde{A_{*} \psi_{h}^{d}}\right)^{-1} \tilde{f} .
$$

Using (3.17) we obtain in $\mathbb{Z}_{N} \times \mathbb{R}_{h_{t}}$

$$
\hat{u}_{h}=\widehat{\psi_{h}^{d}}\left(\widetilde{A_{*} \psi_{h}^{d}}\right)^{-1} \tilde{f} .
$$

The values of $\hat{u}_{h}$ for $\zeta \notin \mathbb{Z}_{N} \times \mathbb{R}_{h_{t}}$ can be determined by using the recurrence relation (3.4) or directly from (3.37) when the discrete Fourier transforms on the right-hand side are defined in $\mathbb{Z} \times \mathbb{R}$ by $\left(N, \frac{2 \pi}{h_{t}}\right)$-periodic extension.

Remark 3.2 In the case of whole space problem the Volterra property of the operator is not used. Our method can be applied also to elliptic problems.

\section{Convergence}

Here we prove convergence results for the collocation starting with the whole space problem

$$
A_{*} u_{h}\left(x_{\kappa}\right)=A_{*} u\left(x_{\kappa}\right), \quad \kappa \in \mathbb{Z}_{N} \times \mathbb{Z} .
$$

Theorem 4.1 Assume the conditions of Theorem 3.5. If $u \in H^{s, \frac{s}{2}}, s>$ $\beta+\frac{3}{2}$, then the collocation solution $u_{h} \in S_{h_{\theta}}^{d_{\theta}} \times S_{h_{t}}^{d_{t}}$ satisfies

$$
\left\|u-u_{h}\right\|_{t, \frac{t}{2}} \leq c h^{\min \{s-t, \bar{d}+1-t\}}\|u\|_{s, \frac{s}{2}}
$$

where $t \leq s, \beta \leq t<\underline{d}+\frac{1}{2}, \underline{d}=\min \left\{d_{\theta}, 2 d_{t}+\frac{1}{2}\right\}, \bar{d}=\min \left\{d_{\theta}, 2 d_{t}+1\right\}$. 
Proof. It suffices to estimate the following three terms

$$
\begin{gathered}
T_{1}=\int_{\mathbb{Z}_{N} \times \mathbb{R}_{h_{t}}}|\underline{\zeta}|^{2 t}\left|\hat{u}(\zeta)-\hat{u}_{h}(\zeta)\right|^{2} \underline{d} \zeta, \\
T_{2}=\int_{\left(\mathbb{Z}_{N} \times \mathbb{R}_{h_{t}}\right)^{\mathrm{c}}}|\underline{\zeta}|^{2 t}\left|\hat{u}_{h}(\zeta)\right|^{2} \underline{d} \zeta, \quad T_{3}=\int_{\left(\mathbb{Z}_{N} \times \mathbb{R}_{h_{t}}\right)^{\mathrm{c}}}|\underline{\zeta}|^{2 t}|\hat{u}(\zeta)|^{2} \underline{d} \zeta,
\end{gathered}
$$

where $\left(\mathbb{Z}_{N} \times \mathbb{R}_{h_{t}}\right)^{c}=(\mathbb{Z} \times \mathbb{R}) \backslash\left(\mathbb{Z}_{N} \times \mathbb{R}_{h_{t}}\right)$. For the last term one easily obtains

$$
T_{3} \leq \operatorname{ch}^{2(s-t)}\|u\|_{s, \frac{s}{2}}^{2}, \quad t \leq s .
$$

Using formula (3.37) for $\hat{u}_{h}$ we get in $\mathbb{Z}_{N} \times \mathbb{R}_{h_{t}}$

$$
\hat{u}-\hat{u}_{h}=\frac{\left.\widetilde{\left(A_{*} \psi_{h}^{d}\right.}-\widehat{A_{*} \psi_{h}^{d}}\right) \hat{u}}{\widehat{A_{*} \psi_{h}^{d}}}-\frac{\widehat{\psi_{h}^{d}}\left(\widetilde{A_{*} u}-\widehat{A_{*} u}\right)}{\widetilde{A_{*} \psi_{h}^{d}}},
$$

which yields by Lemma 3.4 for $\zeta \in \mathbb{Z}_{N} \times \mathbb{R}_{h_{t}}$

$(4.4 a)\left|\hat{u}(\zeta)-\hat{u}_{h}(\zeta)\right| \leq c[h|\zeta|]^{\bar{d}+1-\beta}|\hat{u}(\zeta)|+c|\underline{\zeta}|^{-\beta}\left|\widetilde{A_{*} u}(\zeta)-\widehat{A_{*} u}(\zeta)\right|$,

$$
\left|\hat{u}_{h}(\zeta)\right| \leq c|\hat{u}(\zeta)|+c|\underline{\zeta}|^{-\beta}\left|\widetilde{A_{*} u}(\zeta)-\widehat{A_{*} u}(\zeta)\right| .
$$

Thus we obtain $T_{1} \leq c\left(T_{11}+T_{12}\right)$, where

$$
\begin{aligned}
& T_{11}=\int_{\mathbb{Z}_{N} \times \mathbb{R}_{h_{t}}}|\underline{\zeta}|^{2 t}[h|\underline{\zeta}|]^{2(\bar{d}+1-\beta)}|\hat{u}(\zeta)|^{2} \underline{d} \zeta, \\
& T_{12}=\int_{\mathbb{Z}_{N} \times \mathbb{R}_{h_{t}}}|\underline{\zeta}|^{2(t-\beta)}\left|\widetilde{A_{*} u}(\zeta)-\widehat{A_{*} u}(\zeta)\right|^{2} \underline{d} \zeta .
\end{aligned}
$$

For $T_{11}$ we have

$$
\begin{aligned}
T_{11} & =h^{2(s-t)} \int_{\mathbb{Z}_{N} \times \mathbb{R}_{h_{t}}}[h|\underline{\zeta}|]^{2(\bar{d}+1-\beta-(s-t))}|\underline{\zeta}|^{2 s}|\hat{u}(\zeta)|^{2} \underline{d} \zeta \\
& \leq c h^{2(s-t)}\|u\|_{s, \frac{s}{2}}^{2}, \quad s-t \leq \bar{d}+1-\beta
\end{aligned}
$$

For $\zeta=(n, \eta), \zeta^{\prime}=\left(n^{\prime}, \eta^{\prime}\right)$ we denote $\zeta^{\prime} \sim \zeta$ if $n^{\prime}=n+p N, \eta^{\prime}=\eta+q \frac{2 \pi}{h_{t}}$. By using (2.2) we can conclude

$$
\left|a_{*}\left(\zeta^{\prime}\right)\right| \leq c\left|\underline{\zeta^{\prime}}\right|^{\beta}, \quad \text { for all } \zeta^{\prime} \sim \zeta, \zeta^{\prime} \neq \zeta, \zeta \in \mathbb{Z}_{N} \times \mathbb{R}_{h_{t}} .
$$


Thus we obtain

$$
\begin{aligned}
\left|\widetilde{A_{*} u}(\zeta)-\widehat{A_{*} u}(\zeta)\right|^{2} & =\left|\sum_{\substack{\zeta^{\prime} \sim \zeta \\
\zeta^{\prime} \neq \zeta}} a_{*}\left(\zeta^{\prime}\right) \hat{u}\left(\zeta^{\prime}\right)\right|^{2} \\
& \leq c \sum_{\substack{\zeta^{\prime} \sim \zeta \\
\zeta^{\prime} \neq \zeta}}\left|\underline{\zeta}^{\prime}\right|^{2(\beta-s)} \sum_{\substack{\zeta^{\prime} \sim \zeta \\
\zeta^{\prime} \neq \zeta}}\left|\underline{\zeta}^{\prime}\right|^{2 s}\left|\hat{u}\left(\zeta^{\prime}\right)\right|^{2} .
\end{aligned}
$$

Moreover,

$$
c_{0} h^{-1}\left(|p|+|q|^{\frac{1}{2}}\right) \leq\left|\underline{\zeta^{\prime}}\right| \leq c h^{-1}\left(|p|+|q|^{\frac{1}{2}}\right)
$$

and we get for $s-\beta>\frac{3}{2}$,

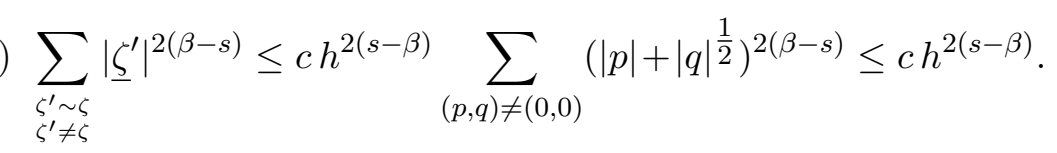

From (4.6), (4.7) it follows

$$
\left|\widetilde{A_{*} u}(\zeta)-\widehat{A_{*} u}(\zeta)\right|^{2} \leq c h^{2(s-\beta)} \sum_{\substack{\zeta^{\prime} \sim \zeta \\ \zeta^{\prime} \neq \zeta}}\left|\underline{\zeta}^{\prime}\right|^{2 s}\left|\hat{u}\left(\zeta^{\prime}\right)\right|^{2}
$$

Using $|\underline{\zeta}|^{2(t-\beta)} \leq c h^{2(\beta-t)}$ for $\zeta \in \mathbb{Z}_{N} \times \mathbb{R}_{h_{t}}, t \geq \beta$ we estimate

$$
\begin{aligned}
T_{12} & \leq c h^{2(\beta-t)} \int_{\mathbb{Z}_{N} \times R_{h_{t}}}\left|\widetilde{A_{*} u}(\zeta)-\widehat{A_{*} u}(\zeta)\right|^{2} \underline{d} \zeta \\
& \leq c h^{2(s-t)} \int_{\mathbb{Z}_{N} \times R_{h_{t}}} \sum_{\substack{\zeta^{\prime} \sim \zeta \\
\zeta^{\prime} \neq \zeta}}\left|\underline{\zeta}^{\prime}\right|^{2 s}\left|\hat{u}\left(\zeta^{\prime}\right)\right|^{2} \underline{d} \zeta \\
& \leq c h^{2(s-t)}\|u\|_{s, \frac{s}{2}}^{2}, \quad t \geq \beta, s>\beta+\frac{3}{2} .
\end{aligned}
$$

For $T_{2}$ we recall the recurrence relations (3.4) for the Fourier coefficients of spline functions $u_{h} \in S_{h_{\theta}}^{d_{\theta}} \times S_{h_{t}}^{d_{t}}$ and obtain

(4.10) $\left|\hat{u}_{h}\left(\zeta^{\prime}\right)\right| \leq c[h|\underline{\zeta}|]^{\bar{d}+1}(1+|p|)^{-\left(d_{\theta}+1\right)}(1+|q|)^{-\left(d_{t}+1\right)}\left|\hat{u}_{h}(\zeta)\right|$. 
Applying (4.10), (3.15) we get for $t<\underline{d}+\frac{1}{2}$

$$
\begin{aligned}
T_{2} & =\int_{\mathbb{Z}_{N} \times R_{h_{t}}} \sum_{\substack{\zeta^{\prime} \sim \zeta \zeta \\
\zeta^{\prime} \neq \zeta}}\left|\underline{\zeta}^{\prime}\right|^{2 t}\left|\hat{u}_{h}\left(\zeta^{\prime}\right)\right|^{2} \underline{d} \zeta \\
& \leq c h^{-2 t} \int_{\mathbb{Z}_{N} \times R_{h_{t}}}[h|\underline{\zeta}|]^{2(\bar{d}+1)} \sum_{(p, q) \in \mathbb{N}_{*}^{2}}\left(p+q^{\frac{1}{2}}\right)^{2 t}(1+p)^{-2\left(d_{\theta}+1\right)} \\
& \leq c h^{2(\bar{d}+1-t)} \int_{\mathbb{Z}_{N} \times R_{h_{t}}}|\underline{\zeta}|^{2(\bar{d}+1)}\left|\hat{u}_{h}(\zeta)\right|^{2} \underline{d} \zeta .
\end{aligned}
$$

For further estimation of $T_{2}$ we use (4.4b), (3.24c) to get $T_{2} \leq c\left(T_{21}+T_{22}\right)$, where

$$
\begin{aligned}
T_{21} & =h^{2(\bar{d}+1-t)} \int_{\mathbb{Z}_{N} \times R_{h_{t}}}|\underline{\zeta}|^{2(\bar{d}+1)}|\hat{u}(\zeta)|^{2} \underline{d} \zeta \\
& =h^{2(s-t)} \int_{\mathbb{Z}_{N} \times R_{h_{t}}}[h \mid \underline{\zeta}]^{2(\bar{d}+1-s)}|\underline{\zeta}|^{2 s}|\hat{u}(\zeta)|^{2} \underline{d} \zeta \\
& \leq c h^{2(s-t)}|| u \|_{s, \frac{s}{2}}^{2}, \quad s \leq \bar{d}+1 .
\end{aligned}
$$

For the other term we obtain by (4.8) for $s-\beta>\frac{3}{2}, \beta \leq \bar{d}+1$,

$$
\begin{aligned}
& \text { (4.12) } T_{22}=h^{2(\bar{d}+1-t)} \int_{\mathbb{Z}_{N} \times R_{h_{t}}}|\underline{\zeta}|^{2(\bar{d}+1-\beta)}\left|\widetilde{A_{*} u}(\zeta)-\widehat{A_{*} u}(\zeta)\right|^{2} \underline{d} \zeta \\
& \leq c h^{2(s-t)} \int_{\mathbb{Z}_{N} \times R_{h_{t}}}[h|\underline{\zeta}|]^{2(\bar{d}+1-\beta)} \sum_{\substack{\zeta^{\prime} \sim \zeta \\
\zeta^{\prime} \neq \zeta}}\left|\underline{\zeta}^{\prime}\right|^{2 s}\left|\hat{u}\left(\zeta^{\prime}\right)\right|^{2} \underline{d} \\
& \leq c h^{2(s-t)} \int_{\mathbb{Z}_{N} \times R_{h_{t}}} \sum_{\substack{\zeta^{\prime} \sim \zeta \\
\zeta^{\prime} \neq \zeta}}\left|\underline{\zeta}^{\prime}\right|^{2 s}\left|\hat{u}\left(\zeta^{\prime}\right)\right|^{2} \underline{d} \zeta \\
& \leq c h^{2(s-t)}\|u\|_{s, \frac{s}{2}}^{2} .
\end{aligned}
$$

Putting the estimates (4.3), (4.5), (4.9), (4.11) and (4.12) together we find the error estimate (4.2).

Remark 4.1 The constant $c$ in the error estimate (4.2) depends on the operator $A_{*}$. More precisely, it depends on the constants $c_{0}, c_{1}$ and $\gamma, \beta$ of the estimates (2.1), (2.2). It will be important, for instance in localization arguments for operators with variable coefficients, that it depends only on these constants and not otherwise on $A_{*}$. 
Next we discuss the collocation problem on the finite interval $[0, T]$. This problem is given by: for $u \in \tilde{H}^{s, \frac{s}{2}}\left(\mathbb{R}_{T}^{2}\right)$ find $u_{h} \in S_{h_{\theta}}^{d_{\theta}} \times \tilde{S}_{h_{t}}^{d_{t}}[0, T]$ such that

$$
L u_{h}\left(x_{\kappa}\right)=L u\left(x_{\kappa}\right), \quad \kappa \in \mathbb{Z}_{N} \times\{1, \cdots, M\} .
$$

Here $M \in \mathbb{N}, h_{t}=\frac{T}{M}$. In order to guarantee that the point values $L u_{h}\left(x_{\kappa}\right)$ are defined we impose in addition to (3.14) the condition

$$
\beta<\underline{d}+\delta-1 .
$$

Observe that in the practical cases where $\delta=1$, (4.14) is valid already by (3.14). In the following proof we will apply the decomposition $L=$ $A+B=A_{*}+B_{*}$. One can easily verify that $A-A_{*}$ defines a bounded operator $A-A_{*}: \tilde{H}^{s, \frac{s}{2}} \rightarrow \tilde{H}^{s-\beta+1, \frac{1}{2}(s-\beta+1)}$. Since $0<\delta \leq 1$ the operator $B_{*}=B+A-A_{*}$ preserves the smoothing property of $B$ such that $B_{*}: \tilde{H}^{s, \frac{s}{2}}\left(\mathbb{R}_{T}^{2}\right) \rightarrow \tilde{H}^{s-\beta+\delta, \frac{s-\beta+\delta}{2}}\left(\mathbb{R}_{T}^{2}\right)$ is bounded. Now the condition (4.14) implies the continuity of the functions $B u_{h}, B_{*} u_{h}$.

Theorem 4.2 Assume (2.1), (3.14), (3.23), (4.14) and that $d_{t}=0$ or $d_{t}=1$. If $u \in \tilde{H}^{s, \frac{s}{2}}\left(\mathbb{R}_{T}^{2}\right), s>\beta+\frac{3}{2}$, then the problem (4.13) has a unique solution for sufficiently small $h$, and we have

$$
\left\|u-u_{h}\right\|_{t, \frac{t}{2} ; T} \leq c h^{\min \{s-t, \bar{d}+1-t\}}\|u\|_{s, \frac{s}{2} ; T},
$$

where $t \leq s, \beta \leq t<\underline{d}+\frac{1}{2}$.

Proof. We look first the case of the operator $A_{*}$ where (4.13) reads

$$
A_{*} u_{h}\left(x_{\kappa}\right)=A_{*} u\left(x_{\kappa}\right), \quad \kappa \in \mathbb{Z}_{N} \times\{1, \cdots, M\} .
$$

For $u \in \tilde{H}^{s, \frac{s}{2}}\left(\mathbb{R}_{T}^{2}\right)$ let $U \in \tilde{H}^{s, \frac{s}{2}}$ be any extension of $u$ such that $u=$ $\left.U\right|_{R_{\theta} \times(-\infty, T)}$. By Theorem 3.5 there exists a unique solution $U_{h} \in S_{h_{\theta}}^{d_{\theta}} \times \tilde{S}_{h_{t}}^{d_{t}}$ of the problem

$$
A_{*} U_{h}\left(x_{\kappa}\right)=A_{*} U\left(x_{\kappa}\right), \quad \kappa \in \mathbb{Z}_{N} \times \mathbb{N} .
$$

By Theorem 3.5, $U_{h}$ is also solution of the corresponding whole space problem. Putting $u_{h}=\left.U_{h}\right|_{R_{\theta} \times(-\infty, T)}$ and using the Volterra property of $A_{*}$ we find that $u_{h}$ is a solution of (4.16). Applying Theorem 4.1 we get

$$
\left\|u-u_{h}\right\|_{t, \frac{t}{2} ; T} \leq\left\|U-U_{h}\right\|_{t, \frac{t}{2}} \leq c h^{s-t}\|U\|_{s, \frac{s}{2}}
$$

which yields

$$
\left\|u-u_{h}\right\|_{t, \frac{t}{2} ; T} \leq c h^{s-t} \inf _{\left.U\right|_{R_{\theta} \times(-\infty, T)=u}}\|U\|_{s, \frac{s}{2}}=c h^{s-t}\|u\|_{s, \frac{s}{2} ; T}
$$


for $\beta \leq t<\underline{d}+\frac{1}{2}, \beta+\frac{3}{2}<s \leq \bar{d}+1$. If $u=0$, it follows from (4.18) that $u_{h}=0$. This implies the existence of a unique solution for (4.16) since it reduces to finite system of equations given by a square matrix. Consider now the general case $L=A+B=A_{*}+B_{*}$. Assume that $u_{h}$ is a solution of (4.13). Introducing $w:=u+A_{*}^{-1} B_{*}\left(u-u_{h}\right)$ we find

$$
A_{*} u_{h}\left(x_{\kappa}\right)=A_{*} w\left(x_{\kappa}\right), \quad \kappa \in \mathbb{Z}_{N} \times\{1, \cdots, M\} .
$$

Moreover

$$
w-u_{h}=\left(I+A_{*}^{-1} B_{*}\right)\left(u-u_{h}\right)=A_{*}^{-1} L\left(u-u_{h}\right)
$$

which yields

$$
u-u_{h}=\left(A_{*}^{-1} L\right)^{-1}\left(w-u_{h}\right)=\left(L^{-1} A_{*}\right)\left(w-u_{h}\right) .
$$

By the mapping properties of $L$ and $A_{*}$ we obtain

$$
\left\|u-u_{h}\right\|_{t, \frac{t}{2} ; T} \leq c\left\|w-u_{h}\right\|_{t, \frac{t}{2} ; T} .
$$

Write $w=u+v, v=A_{*}^{-1} B_{*}\left(u-u_{h}\right)$ and let $u_{h}^{0}, v_{h} \in S_{h_{\theta}}^{d_{\theta}} \times \tilde{S}_{h_{t}}^{d_{t}}[0, T]$ be the collocation solutions such that

$$
\begin{array}{ll}
A_{*} u_{h}^{0}\left(x_{\kappa}\right)=A_{*} u\left(x_{\kappa}\right), & \kappa \in \mathbb{Z}_{N} \times\{1, \cdots, M\}, \\
A_{*} v_{h}\left(x_{\kappa}\right)=A_{*} v\left(x_{\kappa}\right), \quad & \kappa \in \mathbb{Z}_{N} \times\{1, \cdots, M\} .
\end{array}
$$

Since $u_{h}^{0}+v_{h}$ is a collocation solution of $w$ on $\mathbb{R}_{T}^{2}$ with the operator $A_{*}$, the unique solvability of (4.19) implies $u_{h}=u_{h}^{0}+v_{h}$. There holds $w-u_{h}=$ $u-u_{h}^{0}+v-v_{h}$ and by (4.18) we get

$$
\begin{aligned}
\left\|w-u_{h}\right\|_{t, \frac{t}{2} ; T} & \leq\left\|u-u_{h}^{0}\right\|_{t, \frac{t}{2} ; T}+\left\|v-v_{h}\right\|_{t, \frac{t}{2} ; T} \\
& \leq c h^{s-t}\|u\|_{s, \frac{s}{2} ; T}+\left\|v-v_{h}\right\|_{t, \frac{t}{2} ; T} .
\end{aligned}
$$

Putting $\sigma=\min \{\bar{d}+1-t, \delta\}$ and $\rho=\min \{\bar{d}+1, t+\delta\}$ we obtain for the values $\beta \leq t<\underline{d}+\frac{1}{2}, \beta+\frac{3}{2}<s \leq \bar{d}+1$

$$
\begin{aligned}
\left\|v-v_{h}\right\|_{t, \frac{t}{2} ; T} & \leq c h^{\sigma}\left\|A_{*}^{-1} B_{*}\left(u-u_{h}\right)\right\|_{\rho, \frac{\rho}{2} ; T} \\
& \leq c h^{\sigma}\left\|u-u_{h}\right\|_{t, \frac{t}{2} ; T}
\end{aligned}
$$

if additionally $t+\delta>\beta+\frac{3}{2}$. Taking $0<h \leq h_{0}$ small enough we obtain from (4.20-22) the required estimate. Thus we proved (4.15) under the restriction $t>\beta+\frac{3}{2}-\delta$. Assume $\beta \leq t \leq \beta+\frac{3}{2}-\delta$. By (4.14) we have $\beta+\frac{3}{2}<\underline{d}+\frac{1}{2}+\delta$, and we can choose $\tau$ such that $\beta+\frac{3}{2}<\tau<$ $\underline{d}+\frac{1}{2}+\delta, \tau \leq s$. This time we estimate

$$
\left\|v-v_{h}\right\|_{t, \frac{t}{2} ; T} \leq c h^{\tau-t}|| v\left\|_{\tau, \frac{\tau}{2} ; T} \leq c h^{\tau-t}|| u-u_{h}\right\|_{\tau-\delta, \frac{\tau-\delta}{2} ; T} .
$$


Since $\beta+\frac{3}{2}-\delta<\tau-\delta<\underline{d}+\frac{1}{2}$ we can use the already obtained result (4.15) to have

$$
\left\|v-v_{h}\right\|_{t, \frac{t}{2} ; T} \leq c h^{s-t+\delta}\|u\|_{s, \frac{s}{2} ; T} \leq c h^{s-t}\|u\|_{s, \frac{s}{2} ; T} .
$$

Combining (4.21), (4.23) we get (4.15) also for $\beta \leq t \leq \beta+\frac{3}{2}-\delta$. The existence of the unique collocation solution follows by the same argument as in the case of the operator $A_{*}$.

Remark 4.2 In practical applications only choices with low order splines are of interest. To illustrate our results consider some examples. For the single layer heat operator $(\beta=-1)$ we can choose $d_{\theta} \geq 0, d_{t}=0,1$. With piecewise constants we obtain linear convergence for the $L^{2}$-norm, whereas $d_{\theta}=1, d_{t}=0$ gives quadratic convergence rate. Also the choice $d_{\theta}=3, d_{t}=1$ might be of interest, since it yields the rate $O\left(h^{4}\right)$ (assuming sufficient regularity on the solution). For the hypersingular heat operator ( $\beta=1)$ we may use $d_{\theta} \geq 2, d_{t}=1$. With $d_{\theta}=3$ we get the convergence rate $O\left(h^{3}\right)$ for the $L^{2}$ norm and the $H^{1, \frac{1}{2}}$ norm.

Remark 4.3 As in the case of the elliptic problems, we obtain improved convergence results for $H^{t, \frac{t}{2}}$ norms with $t<\beta$ when using splines of even degree. The highest convergence rates for the single layer heat operator are obtained in the $H^{-2,-1}$ norm, with $d_{\theta}=d_{t}=0: O\left(h^{3}\right)$ and with $d_{\theta}=2, d_{t}=1: O\left(h^{5}\right)$. For the hypersingular operator, we obtain with $d_{\theta}=2, d_{t}=1: O\left(h^{3}\right)$ in the $L^{2}$ norm.

More generally, we have the following result.

Theorem 4.3 Assume the conditions of Theorem 4.2 with $0<\delta \leq 1$ in (2.1g) and that $d_{\theta}$ is even. Then for $s>\beta+\frac{3}{2}, t<\underline{d}+\frac{1}{2}, t \leq s$, we have for sufficiently small $h$,

$$
\left\|u-u_{h}\right\|_{t, \frac{t}{2} ; T} \leq c h^{\alpha}\|u\|_{s, \frac{s}{2} ; T}
$$

with $\alpha=\min \{s-t, s-\beta, \bar{d}+1-t, \tilde{d}+2-\beta, \bar{d}+1-\beta+\delta\}$, where $\tilde{d}=\min \left\{d_{\theta}, 2 d_{t}\right\}$.

Proof. We first note that the perturbation operator $B$ (or equivalently $B_{*}$ ) affects the improved convergence result (4.24) only if $\delta<1$ since we have $\tilde{d} \leq \bar{d}$.

The proof will now be done in three steps. The main observation is that for even $d_{\theta}$, the consistency estimate (3.24c) of Lemma 3.4 can be improved:

$$
\left|\widetilde{A_{*} \psi_{h}^{d}}(\zeta)-\widehat{A_{*} \psi_{h}^{d}}(\zeta)\right| \leq c[h|\underline{\zeta}|]^{\tilde{d}+2-\beta}\left|\widetilde{A_{*} \psi_{h}^{d}}(\zeta)\right| .
$$


The proof of (4.25) will be the first step.

We consider the function $T(\zeta)$ defined in (3.25) in the proof of Lemma 3.4:

$$
T(\zeta)=\sum_{(p, q) \neq(0,0)} a_{*}\left(\zeta_{p, q}\right)(-1)^{\kappa_{p, q}}\left[\frac{n}{n+p N}\right]^{d_{\theta}+1}\left[\frac{\eta}{\eta+q \frac{2 \pi}{h_{t}}}\right]^{d_{t}+1} .
$$

It suffices to consider the case $n \neq 0$. Defining $\xi=\frac{n}{N} \in\left[-\frac{1}{2}, \frac{1}{2}\right]$ and $\tau=h_{t} \eta \in[-\pi, \pi]$ and using the homogeneity of $a$, we have then $T(\zeta)=$ $N^{\beta} F(\xi, \tau)$ with

$$
F(\xi, \tau)=\sum_{(p, q) \neq(0,0)} a(\xi+p, \nu(\tau+2 \pi q))(-1)^{\kappa_{p, q}}\left[\frac{\xi}{\xi+p}\right]^{d_{\theta}+1}\left[\frac{\tau}{\tau+2 \pi q}\right]^{d_{t}+1} .
$$

By (3.15), this series converges together with all its derivatives and therefore $F$ is a $C^{\infty}$ function on $\left[-\frac{1}{2}, \frac{1}{2}\right] \times[-\pi, \pi]$. Since $(-1)^{\kappa_{p, q}}=(-1)^{\kappa_{-p, q}}$ and $a(-x, y)=a(x, y)$, we have

$$
\begin{aligned}
F(-\xi, \tau) & =\sum_{(p, q) \neq(0,0)} a(-\xi-p, \nu(\tau+2 \pi q))(-1)^{\kappa_{p, q}} \\
\cdot\left[\frac{-\xi}{-\xi-p}\right]^{d_{\theta}+1}\left[\frac{\tau}{\tau+2 \pi q}\right]^{d_{t}+1} & \\
& =F(\xi, \tau) .
\end{aligned}
$$

Therefore there exists a function $G \in C^{\infty}\left(\left[-\frac{1}{4}, \frac{1}{4}\right] \times[-\pi, \pi]\right)$ such that $F(\xi, \tau)=G\left(\xi^{2}, \tau\right)$. Using the fact that $h|\underline{\zeta}|=|\xi|+(\nu|\tau|)^{\frac{1}{2}}$, we see that the estimate $(3.24 \mathrm{c})$ means

$$
|F(\xi, \tau)| \leq c\left(\xi^{2}+|\tau|\right)^{(\bar{d}+1) / 2} .
$$

Let $G(x, y)=P_{d}(x, y)+O\left(\left(x^{2}+y^{2}\right)^{(d+1) / 2}\right)$ be the Taylor expansion of $G$ at the origin where

$$
P_{d}(x, y)=\sum_{k=0}^{d} \alpha_{k} x^{k} y^{d-k}
$$

is the lowest-order non-zero term. From the estimate (4.26) follows that

$$
d \geq \frac{\bar{d}+1}{2}=\min \left\{\frac{d_{\theta}+1}{2}, d_{t}+1\right\} .
$$

Since $d$ is an integer and $d_{\theta}$ is even, this implies immediately

$$
d \geq \min \left\{\frac{d_{\theta}+2}{2}, d_{t}+1\right\}=\frac{\tilde{d}}{2}+1 .
$$


Thus (4.26) can be improved to

$$
|F(\xi, \tau)| \leq c\left(\xi^{2}+|\tau|\right)^{(\tilde{d}+2) / 2},
$$

hence

$$
|T(\zeta)| \leq c h^{-\beta}[h|\underline{\zeta}|]^{\tilde{d}+2},
$$

and thus the estimate (4.25) is shown.

As a second step, we reconsider the proof of Theorem 4.1. This means that we want to show the estimate (4.24) for the case where the perturbation $B_{*}$ is absent. Then we have to look at the error for the finite interval $\mathbb{R}_{T}^{2}$. The following estimates from the proof of Theorem 4.1 are already compatible with (4.24):

$$
\begin{aligned}
T_{12} & \leq c h^{2 \min \{s-t, s-\beta\}}\|u\|_{s, \frac{s}{2}}^{2} ; \\
T_{21} & \leq c h^{2 \min \{s-t, \bar{d}+1-t\}}\|u\|_{s, \frac{s}{2}}^{2} ; \\
T_{22}+T_{3} & \leq c h^{2(s-t)}\|u\|_{s, \frac{s}{2}}^{2} .
\end{aligned}
$$

The only term that needs improvement is $T_{11}$. Using (4.25), we can improve the estimate (4.4a) to

$$
\left|\hat{u}(\zeta)-\hat{u}_{h}(\zeta)\right| \leq c[h|\underline{\zeta}|]^{\tilde{d}+2-\beta}|\hat{u}(\zeta)|+c|\underline{\zeta}|^{-\beta}\left|\widetilde{A_{*} u}(\zeta)-\widehat{A_{*} u}(\zeta)\right| .
$$

We change therefore the definition of $T_{11}$ to

$$
T_{11}=\int_{\mathbb{Z}_{N} \times \mathbb{R}_{h_{t}}}|\zeta|^{2 t}[h|\underline{\zeta}|]^{2(\tilde{d}+2-\beta)}|\hat{u}(\zeta)|^{2} \underline{d} \zeta,
$$

and we see that now

$$
T_{11} \leq c h^{2(s-t)}\|u\|_{s, \frac{s}{2}}^{2}, \quad \text { for } s-t \leq \tilde{d}+2-\beta .
$$

This completes the proof of (4.24) for this case. As for the finite interval $\mathbb{R}_{T}^{2}$, we obtain with exactly the same argument as in (4.18), that (4.24) is valid, even for $\delta=1$ which corresponds to the case where the perturbation is not present.

In the final step, we incorporate the perturbation $B_{*}$.

Using the notations of the proof of Theorem 4.2, we obtain, as we have just shown,

$$
\left\|u-u_{h}^{0}\right\|_{t, \frac{t}{2} ; T} \leq c h^{\min \{s-t, s-\beta, \bar{d}+1-t, \tilde{d}+2-\beta\}}\|u\|_{s, \frac{s}{2} ; T},
$$

and taking $t=\beta, s=\bar{d}+1$ in (4.23),

$$
\left\|v-v_{h}\right\|_{\beta, \frac{\beta}{2} ; T} \leq c h^{\bar{d}+1+\delta-\beta}\|u\|_{\bar{d}+1, \frac{\bar{d}+1}{2} ; T} .
$$


The assertion (4.24) follows now by combining (4.20), (4.21), (4.29) and (4.30).

Let us now consider the estimate (4.24) in more detail. The first observation is that if the parameters $t$ and $s$ both lie in the range allowed by Theorem 4.2, namely $\beta \leq t<\underline{d}+\frac{1}{2}, \beta+\frac{3}{2}<s \leq \bar{d}+1$, then (4.24) coincides with (4.15). Further, in order to obtain a higher convergence rate than allowed by (4.25), one has to take values $t$ and $s$ satisfying $t<\beta$ and $s>\bar{d}+1$ simultaneously. This implies that an improved estimate occurs if and only if there holds

$$
\bar{d}+1-\beta<\tilde{d}+2-\beta, \quad \text { i. e. } 0<\tilde{d}-\bar{d}+1 .
$$

On the other hand, we have $\tilde{d} \leq \bar{d}$, and thus (4.31) becomes $0<\tilde{d}-\bar{d}+1 \leq$ 1. But since $\tilde{d}$ and $\bar{d}$ are integers, (4.31) holds if and only if $\tilde{d}=\bar{d}$. As we consider only the two values $d_{t}=0$ and $d_{t}=1$, all the cases where an improvement occurs are contained in the following list:

$$
d_{\theta}=0, d_{t}=0 ; \quad d_{\theta}=0, d_{t}=1 ; \quad d_{\theta}=2, d_{t}=1 .
$$

Finally, we can write the improved estimates in a symmetric form; we simultaneously take $t=\beta-\tau$ and $s=\bar{d}+1+\tau, \tau>0$.

Now Theorem 4.3 takes the following equivalent but more explicit form.

Theorem 4.4 Assume the conditions of Theorem 4.2 with $0<\delta \leq 1$ in (2.1 $\mathrm{g}$ ) and that $d_{\theta}$ is even. Then in addition to the optimal order result (4.15), there holds the suboptimal estimate

$$
\left\|u-u_{h}\right\|_{\beta-\tau, \frac{\beta-\tau}{2} ; T} \leq c h^{\bar{d}+1+\tau-\beta}\|u\|_{\bar{d}+1+\tau, \frac{\bar{d}+1+\tau}{2} ; T},
$$

if $0<\tau \leq \delta$ and $\left(d_{\theta}, d_{t}\right)$ satisfies one of the conditions (4.32). The highest convergence rate is $\bar{d}+1-\beta+\delta$. The order of the improvement is 1 .

Remark 4.4 If the order $\beta$ of the operator $L$ is positive, we obtain an improved convergence with respect to the non-negative norms $\left\|u-u_{h}\right\|_{\beta-\tau, \frac{\beta-\tau}{2} ; T}, 0 \leq$ $\tau \leq \min \{\beta, \delta\}$. If $\beta \leq 0$, we obtain improved convergence results only for some norms of negative order. But such results are still of interest as in the elliptic case. An application is given below.

Example 4.1 Consider the single layer heat potential which is given for $x \notin \Gamma$ by

$$
\Phi(x, t)=\int_{0}^{t} \int_{-\frac{1}{2}}^{\frac{1}{2}} u\left(\phi, t^{\prime}\right) E\left(x-x(\phi), t-t^{\prime}\right)\left|x^{\prime}(\phi)\right| d \phi d t^{\prime} .
$$


For the notations see Example 2.1. Assume that we have determined the collocation solution $u_{h}(\phi, t),(\phi, t) \in \mathbb{R}_{T}^{2}$ by using the single layer heat equation. Then we have the approximate heat potential for $0<t \leq T$

$$
\Phi_{h}(x, t)=\int_{0}^{t} \int_{-\frac{1}{2}}^{\frac{1}{2}} u_{h}\left(\phi, t^{\prime}\right) E\left(x-x(\phi), t-t^{\prime}\right)\left|x^{\prime}(\phi)\right| d \phi d t^{\prime} .
$$

Applying (4.33) with $\beta=-1, \delta=1$ we get

$$
\begin{array}{rr}
\left|\Phi(x, t)-\Phi_{h}(x, t)\right| \leq c\left\|u-u_{h}\right\|_{-2,-1 ; T} \leq c h^{\bar{d}+3}\|u\|_{\bar{d}+2, \frac{\bar{d}+2}{2} ; T}, \\
c=c(x, t) .
\end{array}
$$

Thus we have the following improved rates for the heat potential

$\left|\Phi(x, t)-\Phi_{h}(x, t)\right| \leq c \begin{cases}h^{3}, & \text { if } d_{\theta}=d_{t}=0 \text { or } d_{\theta}=0 \text { and } d_{t}=1, \\ h^{5}, & \text { if } d_{\theta}=2 \text { and } d_{t}=1 .\end{cases}$

The maximal order of improvement is 1 .

\section{Appendix. Stability: Splines of even degree}

We want to show the positivity estimate (3.24b) under the hypotheses (3.14c) or $(3.14 \mathrm{~d})$.

We assume first that the degree $d_{t}$ is even and $d_{\theta}$ is odd. In addition, we assume that the principal symbol has the special form (2.3) with $-2<\beta<$ 2 .

In the sum

$$
a_{*}^{c}(\zeta)=\sum_{p, q} a_{*}\left(\zeta_{p, q}\right)(-1)^{\kappa_{p, q}}\left[\frac{n}{n+p N}\right]^{d_{\theta}+1}\left[\frac{\eta}{\eta+q \frac{2 \pi}{h_{t}}}\right]^{d_{t}+1} \widehat{\psi_{h}^{d}}(\zeta)
$$

we have $(-1)^{\kappa_{p, q}}=(-1)^{q}$. Since both $\operatorname{Re} a_{*}(\xi, \eta)$ and $\widehat{\psi_{h}^{d}}(\xi, \eta)$ are even functions of $\xi$ and $\eta$, we may assume that $n$ and $\eta$ in (5.1) are positive. For the same reason, it suffices to consider the sum

$$
a_{*}^{++}(\zeta)=\sum_{p \geq 0, q \geq 0} a_{*}\left(\zeta_{p, q}\right)(-1)^{\kappa_{p, q}}\left[\frac{n}{n+p N}\right]^{d_{\theta}+1}\left[\frac{\eta}{\eta+q \frac{2 \pi}{h_{t}}}\right]^{d_{t}+1} \widehat{\psi_{h}^{d}}(\zeta)
$$

If we can show that this has positive real part, then the 3 remaining sums over $p \geq 0, q<0$, over $p<0, q \geq 0$, and over $p<0, q<0$ will have positive real part, too, since they can be written as sums of the form (5.2) 
(with $n$ replaced by $N-n$ and/or $\eta$ replaced by $\frac{2 \pi}{h_{t}}-\eta$ ).

We write

$$
a_{*}^{++}(\zeta)=\sum_{p=0}^{\infty}\left[\frac{n}{n+p N}\right]^{d_{\theta}+1} \widehat{\psi_{h}^{d}}(\zeta) \sum_{q=0}^{\infty} S_{p, 2 q}
$$

with

$$
\begin{aligned}
S_{p, 2 q}= & \left.a_{\beta}\left(n+p N, \eta+2 q \frac{2 \pi}{h_{t}}\right)\right)\left[\frac{\eta}{\eta+2 q \frac{2 \pi}{h_{t}}}\right]^{d_{t}+1} \\
& -a_{\beta}\left(n+p N, \eta+(2 q+1) \frac{2 \pi}{h_{t}}\right)\left[\frac{\eta}{\eta+(2 q+1) \frac{2 \pi}{h_{t}}}\right]^{d_{t}+1}
\end{aligned}
$$

We shall show now that for $\xi>0$ the positive function

$$
\tau \mapsto g(\tau)=\operatorname{Re} a_{\beta}(\xi, \tau)\left[\frac{1}{\tau}\right]^{d_{t}+1} \quad \text { has a negative derivative for } \tau>0 \text {. }
$$

According to the homogeneity assumption (2.3), we can simplify (5.4) by assuming that $\alpha|\xi|^{2}=1$, and we compute then with $\tau=\tan \phi, \phi \in\left(0, \frac{\pi}{2}\right)$

$$
\begin{aligned}
g^{\prime}(\tau) & =\operatorname{Re}\left[\frac{\partial}{\partial \tau}(1+\mathrm{i} \tau)^{\frac{\beta}{2}} \tau^{-d_{t}-1}\right] \\
& =|1+\mathrm{i} \tau|^{\frac{\beta}{2}-1} \tau^{-d_{t}-2} \operatorname{Re}\left[\left(\mathrm{i} \beta \tau / 2-\left(d_{t}+1\right)(1+\mathrm{i} \tau)\right) \mathrm{e}^{\mathrm{i} \phi\left(\frac{\beta}{2}-1\right)}\right]
\end{aligned}
$$

This can be written in the following two equivalent forms:

$$
\begin{aligned}
& g^{\prime}(\tau)|1+\mathrm{i} \tau|^{-\frac{\beta}{2}+1} \tau^{d_{t}+2} \\
& \quad=\frac{-1}{\cos \phi}\left\{\left(d_{t}+1\right) \cos \phi \frac{\beta}{2}+\frac{\beta}{2} \sin \phi \sin \phi\left(\frac{\beta}{2}-1\right)\right\} \\
& \quad=\frac{-1}{\cos \phi}\left\{\left(d_{t}+1-\frac{\beta}{2}\right) \cos \phi \frac{\beta}{2}+\frac{\beta}{2} \cos \phi \cos \phi\left(\frac{\beta}{2}-1\right)\right\}
\end{aligned}
$$

The first expression on the right hand side is easily seen to be negative for $\beta \in[-2,0]$ and the second for $\beta \in[0,2]$. Thus (5.4) is shown.

If we set $\xi=n+p N$ and $\tau=\eta+2 q \frac{2 \pi}{h_{t}}$ in (5.4), we obtain immediately that $\operatorname{Re} S_{p, 2 q}=\eta^{d_{t}+1}\left(g(\tau)-g\left(\tau+\frac{2 \pi}{h_{t}}\right)\right)>0$ for all $p, q \geq 0$.

It remains to estimate

$$
\begin{aligned}
S_{0,0} & =\left(\alpha n^{2}+\mathrm{i} \eta\right)^{\beta / 2}-\left(\alpha n^{2}+\mathrm{i}\left(\eta+\frac{2 \pi}{h_{t}}\right)\right)^{\beta / 2}\left[\frac{\eta}{\eta+\frac{2 \pi}{h_{t}}}\right]^{d_{t}+1} \\
& =h_{t}^{-\beta / 2}\left(\left(\xi^{2}+\mathrm{i} \tau\right)^{\beta / 2}-\left(\xi^{2}+\mathrm{i}(\tau+2 \pi)\right)^{\beta / 2}\left[\frac{\tau}{\tau+2 \pi}\right]^{d_{t}+1}\right),
\end{aligned}
$$

where we set $\xi^{2}=\alpha n^{2} h_{t}$ and $\tau=\eta h_{t}$. With (3.23) we see that

$$
\left(\xi^{2}, \tau\right) \in\left[0, \frac{\alpha}{4 \nu}\right] \times[0, \pi]=: Q
$$


We see that $f\left(\xi^{2}, \tau\right)=h_{t}{ }^{\beta / 2} \operatorname{Re} S_{0,0}$ is a continuous function on $Q \backslash\{(0,0)\}$ and positive there, as we have seen before. At the origin, we obtain with $d_{t}+1>\beta / 2$

$\liminf \left|\xi^{2}+\mathrm{i} \tau\right|^{-\beta / 2} f\left(\xi^{2}, \tau\right)=\liminf \left|\xi^{2}+\mathrm{i} \tau\right|^{-\beta / 2} \operatorname{Re}\left(\xi^{2}+\mathrm{i} \tau\right)^{\beta / 2}>0$.

This implies that on $Q \backslash\{(0,0)\}, f\left(\xi^{2}, \tau\right) \geq c\left|\xi^{2}+\mathrm{i} \tau\right|^{\beta / 2}$ with some $c>0$. Hence

$$
\operatorname{Re} S_{0,0} \geq c h_{t}{ }^{-\beta / 2}\left|\xi^{2}+\mathrm{i} \tau\right|^{\beta / 2} \geq c_{0}|\underline{\zeta}|^{\beta} .
$$

Thus (3.24b) is shown for this case.

As a second case, we assume now that $d_{t}$ is odd and $d_{\theta}$ is even. The principal symbol has the special form (2.3) with $-2<\beta<2$. In addition, we have $-\left(d_{\theta}+1\right) \leq \beta \leq d_{\theta}+1$, which is restrictive only if $d_{\theta}=0$, or if $|\beta|>1$. We have again the formula (5.2), but now $(-1)^{\kappa_{p, q}}=(-1)^{p}$. We can therefore write

$$
a_{*}^{++}(\zeta)=\sum_{p=0}^{\infty}\left[\frac{\eta}{\eta+q \frac{2 \pi}{h_{t}}}\right]^{d_{t}+1} \widehat{\psi_{h}^{d}}(\zeta) \sum_{q=0}^{\infty} S_{2 p, q}
$$

where once again the terms in the second sum are differences

$$
\begin{aligned}
S_{2 p, q}= & \left.a_{\beta}\left(n+2 p N, \eta+q \frac{2 \pi}{h_{t}}\right)\right)\left[\frac{n}{n+2 p N}\right]^{d_{\theta}+1} \\
& -a_{\beta}\left(n+(2 p+1) N, \eta+q \frac{2 \pi}{h_{t}}\right)\left[\frac{n}{n+(2 p+1) N}\right]^{d_{\theta}+1} .
\end{aligned}
$$

Thus with $\xi=n+2 p N, \tau=\eta+q \frac{2 \pi}{h_{t}}$ and $g(\xi)=\operatorname{Re} a_{\beta}(\xi, \tau) \xi^{-d_{\theta}-1}$, we have

$$
\operatorname{Re} S_{2 p, q}=n^{d_{\theta}+1}(g(\xi)-g(\xi+N)) .
$$

We will finish the proof for this second case as above by showing that $g$ is a decreasing function. By writing $\sigma=\frac{\alpha|\xi|^{2}}{\tau}$, we find

$$
\begin{aligned}
g(\xi) & =\operatorname{Re} \xi^{-\left(d_{\theta}+1\right)}\left(\alpha|\xi|^{2}+\mathrm{i} \tau\right)^{\beta / 2} \\
& =\alpha^{\left(d_{\theta}+1\right) / 2} \tau^{\left(\beta-d_{\theta}-1\right) / 2} \operatorname{Re} \sigma^{-\left(d_{\theta}+1\right) / 2}(\sigma+\mathrm{i})^{\beta / 2} .
\end{aligned}
$$

It remains therefore to show that the function

$$
\sigma \mapsto h(\sigma)=\operatorname{Re} \sigma^{-\left(d_{\theta}+1\right) / 2}(\sigma+\mathrm{i})^{\beta / 2} \text { has a negative derivative for } \sigma>0 .
$$

Let $\sigma=\cot \phi, \phi \in\left(0, \frac{\pi}{2}\right)$. We find

$$
h^{\prime}(\sigma)=\sigma^{-\frac{d_{\theta}+3}{2}}|\sigma+\mathrm{i}|^{\frac{\beta}{2}-1} \operatorname{Re}\left[\left(\frac{\beta \sigma}{2}-\frac{d_{\theta}+1}{2}(\sigma+\mathrm{i})\right) \mathrm{e}^{\mathrm{i} \phi\left(\frac{\beta}{2}-1\right)}\right] .
$$


In order to see that this is negative, we have to use a slightly different substitution from the one used in the first case above. Thus we further introduce $\psi \in(0, \phi)$ by $-2 \psi=\phi\left(\frac{\beta}{2}-1\right)$ and $\delta=\tan \psi$. We have then

$$
0<\sigma \delta<1 \quad \text { and } \cos \phi\left(\frac{\beta}{2}-1\right)=\frac{1-\delta^{2}}{1+\delta^{2}}, \sin \phi\left(\frac{\beta}{2}-1\right)=\frac{-2 \delta}{1+\delta^{2}}
$$

Hence we compute

$$
\begin{aligned}
& h^{\prime}(\sigma) \sigma^{\frac{d_{\theta}+3}{2}}|\sigma+\mathrm{i}|^{-\frac{\beta}{2}+1} \\
& \quad=\frac{-1}{2\left(1+\delta^{2}\right)}\left\{\left(d_{\theta}+1-\beta\right) \sigma\left(1-\delta^{2}\right)+2\left(d_{\theta}+1\right) \delta\right\} \\
& \quad=\frac{-1}{2\left(1+\delta^{2}\right)}\left\{\left(d_{\theta}+1-\beta\right)(\sigma+\delta(1-\sigma \delta))+\left(d_{\theta}+1+\beta\right) \delta\right\} .
\end{aligned}
$$

This last formulation shows clearly that $h^{\prime}(\sigma)<0$.

We have thus shown that $\operatorname{Re} a_{*}^{c}(\zeta)>\operatorname{Re} S_{0,0} \widehat{\psi_{h}^{d}}(\zeta)>0$. The estimate (5.5) for $\operatorname{Re} S_{0,0}$ follows by the same argument as above in the first case.

For the third and final case we assume that (3.14d) holds, i. e. both $d_{t}$ and $d_{\theta}$ are even. In the sum (5.2), we have now $(-1)^{\kappa_{p, q}}=(-1)^{p+q}$. The sum is therefore alternating in both directions, and we group the summands by four:

$$
a_{*}^{++}(\zeta)=\widehat{\psi_{h}^{d}}(\zeta) \sum_{p, q=0}^{\infty} S_{2 p, 2 q}
$$

where we define

$$
\begin{aligned}
& S_{2 p, 2 q}= \\
& \quad a_{\beta}\left(n+2 p N, \eta+2 q \frac{2 \pi}{h_{t}}\right)\left[\frac{n}{n+2 p N}\right]^{d_{\theta}+1}\left[\frac{\eta}{\eta+2 q \frac{2 \pi}{h_{t}}}\right]^{d_{t}+1} \\
& -a_{\beta}\left(n+(2 p+1) N, \eta+2 q \frac{2 \pi}{h_{t}}\right)\left[\frac{n}{n+(2 p+1) N}\right]^{d_{\theta}+1}\left[\frac{\eta}{\eta+2 q \frac{2 \pi}{h_{t}}}\right]^{d_{t}+1} \\
& -a_{\beta}\left(n+2 p N, \eta+(2 q+1) \frac{2 \pi}{h_{t}}\right)\left[\frac{n}{n+2 p N}\right]^{d_{\theta}+1}\left[\frac{\eta}{\eta+(2 q+1) \frac{2 \pi}{h_{t}}}\right]^{d_{t}+1} \\
& +a_{\beta}\left(n+(2 p+1) N, \eta+(2 q+1) \frac{2 \pi}{h_{t}}\right)\left[\frac{n}{n+(2 p+1) N}\right]^{d_{\theta}+1} \\
& \quad \times\left[\frac{\eta}{\eta+(2 q+1) \frac{2 \pi}{h_{t}}}\right]^{d_{t}+1}
\end{aligned}
$$


With $\xi=n+2 p N, \tau=\eta+2 q \frac{2 \pi}{h_{t}}$ and $g(\xi, \tau)=\operatorname{Re} a_{\beta}(\xi, \tau) \xi^{-\left(d_{\theta}+1\right)}$ $\cdot \tau^{-\left(d_{t}+1\right)}$, we have

$\operatorname{Re} S_{2 p, 2 q}$

$$
\begin{aligned}
& =n^{d_{\theta}+1} \eta^{d_{t}+1}\left(g(\xi, \tau)-g(\xi+N, \tau)-g\left(\xi, \tau+\frac{2 \pi}{h_{t}}\right)+g\left(\xi+N, \tau+\frac{2 \pi}{h_{t}}\right)\right) \\
& =n^{d_{\theta}+1} \eta^{d_{t}+1} \int_{\xi}^{\xi+N} \int_{\tau}^{\tau+\frac{2 \pi}{h_{t}}} \frac{\partial^{2} g(\xi, \tau)}{\partial \xi \partial \tau} d \tau d \xi .
\end{aligned}
$$

As above in the first two cases, we will be able to conclude the positivity of $\operatorname{Re} S_{2 p, 2 q}$ and therefore the estimate (3.24b) for $a_{*}^{c}(\zeta)$ if we show that the mixed derivative

$$
\frac{\partial^{2}}{\partial \xi \partial \tau} g(\xi, \tau) \quad \text { is positive for } \xi, \tau>0 .
$$

We set $\sigma=\alpha \xi^{2}, d_{1}=\left(d_{\theta}+1\right) / 2, d_{2}=d_{t}+1$, and $\gamma=\beta / 2$, and we compute

$$
\begin{aligned}
h(\sigma, \tau):=\frac{\partial^{2}}{\partial \sigma \partial \tau} \sigma^{-d_{1}} \tau^{-d_{2}}(\sigma+\mathrm{i} \tau)^{\gamma} & \\
= & \sigma^{-d_{1}-1} \tau^{-d_{2}-1}(\sigma \\
& +\mathrm{i} \tau)^{\gamma-2}\left\{d_{2}\left(d_{1}-\gamma\right) \sigma^{2}+d_{1}\left(\gamma-d_{2}\right) \tau^{2}\right. \\
& \left.+\mathrm{i}\left(2 d_{1} d_{2}-\left(d_{1}+d_{2}\right) \gamma+\gamma(\gamma-1)\right) \sigma \tau\right\} .
\end{aligned}
$$

We can now assume that $\sigma=1$, and we set $\tau=\tan \phi, x=\cos \phi=$ $|1+\mathrm{i} \tau|^{-1}$. We obtain

$$
\begin{aligned}
p:= & \tau^{d_{2}+1}|1+\mathrm{i} \tau|^{-\gamma} \operatorname{Re} h(1, \tau) \\
(5.8)= & \cos ^{2} \phi \cos (\gamma-2) \phi\left\{d_{2}\left(d_{1}-\gamma\right)+d_{1}\left(\gamma-d_{2}\right) \tau^{2}\right\} \\
& -\sin \phi \cos \phi \sin (\gamma-2) \phi\left\{2 d_{1} d_{2}-\left(d_{1}+d_{2}\right) \gamma+\gamma(\gamma-1)\right\} .
\end{aligned}
$$

Now we use the identities

$$
\begin{aligned}
\cos (\gamma-2) \phi= & 2 \cos \phi \cos (\gamma-1) \phi-\cos \gamma \phi \\
= & \left(4 \cos ^{2} \phi-1\right) \cos \gamma \phi-2 \cos \phi \cos (\gamma+1) \phi \\
\sin \phi \cos \phi \sin (\gamma-2) \phi= & \frac{1}{2}(-\cos \gamma \phi+\cos 2 \phi \cos (\gamma-2) \phi) \\
= & \left(4 \cos ^{4} \phi-3 \cos ^{2} \phi\right) \cos \gamma \phi \\
& +\left(-2 \cos ^{3} \phi+\cos \phi\right) \cos (\gamma+1) \phi
\end{aligned}
$$

and transform (5.8) into

$$
p=q_{1}(x) \cos \gamma \phi+q_{2}(x) \cos (\gamma+1) \phi
$$


with

$$
\begin{aligned}
& q_{1}(x)=-4 \gamma(\gamma-1) x^{4}+\left(2\left(d_{1}-d_{2}\right) \gamma+3 \gamma(\gamma-1)\right) x^{2}+d_{1}\left(d_{2}-\gamma\right) \\
& q_{2}(x)=2 \gamma(\gamma-1) x^{3}+\left(\left(d_{2}-d_{1}\right) \gamma-\gamma(\gamma-1)\right) x .
\end{aligned}
$$

From this form of the expression $p$, it would not be too hard to show its positivity for negative $\gamma$ and sufficiently large $d_{1}, d_{2}$, while for positive $\gamma$ one would use a different expression involving $\cos (\gamma-1) \phi$. We shall, however, concentrate now on the case at hand, namely $\beta=-1$ and $d_{t}=0$, that is $\gamma=-1 / 2$ and $d_{2}=1$, and we want to show that $p>0$ holds for all $x \in(0,1)$ and all $d_{1} \geq 1 / 2$.

We have in this case

$$
p=\left(q_{1}(x)+q_{2}(x)\right) \cos \frac{\phi}{2}=: \frac{q(x)}{4} \cos \frac{\phi}{2}
$$

with the polynomial

$$
q(x)=-12 x^{4}+6 x^{3}+\left(13-4 d_{1}\right) x^{2}+\left(2 d_{1}-5\right) x+6 d_{1} .
$$

The partial derivative of $q(x)$ with respect to $d_{1}$ is $(x+1)(6-4 x)$ which is positive. It remains to show that $q(x)$ is positive for $d_{1}=1 / 2$ in order to obtain its positivity for all $d_{1} \geq 1 / 2$.

For $d_{1}=1 / 2$, we find

$$
\begin{aligned}
q(x) & =-12 x^{4}+6 x^{3}+11 x^{2}-4 x+3 \\
& =6(1+2 x)(1-x) x^{2}+x^{2}+(2 x-1)^{2}+2
\end{aligned}
$$

which is clearly positive for all $x \in(0,1)$. This concludes the proof for the third case.

\section{References}

1. Abramowitz, M., Stegun, I.A. (1971): Handbook of Mathematical Functions with Formulas, Graphs and Mathematical Tables. GPO, Washington

2. Arnold, D.N., Noon, P.J. (1989): Coercivity of the single layer heat potential. J. Comput. Math. 7, 100-104

3. Arnold, D.N., Saranen, J. (1984): On the asymptotic convergence of spline collocation methods for partial differential equations. SIAM J. Numer. Anal. 21, 459-472

4. Arnold, D.N., Wendland, W.L. (1983): On the asymptotic convergence of collocation methods Math. Comp. 41, 349-381

5. Arnold, D.N., Wendland, W.L. (1985): The convergence of spline collocation for strongly elliptic equations on curves. Numer. Math. 47, 317-343

6. Bramble, J.H., Hilbert, S.R. (1970): Estimation of linear functionals on Sobolev spaces with application to Fourier transforms and spline interpolation. SIAM J. Numer. Anal. 7, 112-124

7. Brown, R. (1988): Layer potentials and boundary value problems for the heat equation on Lipschitz cylinders. University of Minnesota, Ph.D Thesis 
8. Costabel, M. (1990): Boundary integral operators for the heat equation. Integral Equations. Operator Theory 13, 498-552

9. Costabel, M., McLean, W. (1992): Spline collocation for strongly elliptic equations on the torus Numer. Math. 62, 511-538

10. Costabel, M., Onishi, K., Wendland, W.L. (1987): A boundary element collocation method for the Neumann problem of the heat equation. In: Engl, H.W., Groetsch, C.W. (eds.) Inverse and Ill-Posed Problems. Academic Press, New York, pp. 369-384

11. Costabel, M., Penzel, F., Schneider, R. (1992): Error analysis of boundary element collocation method for a screen problem in $\mathbb{R}^{3}$. Math. Comp. 58, 575-586

12. Fabes, E.B., Riviere, N.M. (1979): Dirichlet and Neumann problems for the heat equation in $C^{1}$-cylinders. Proceedings of Symposia in Pure Mathematics, vol. XXXV, Part 2, pp. 179-196

13. Friedman, A. (1983): Partial differential equations of parabolic type. Robert E. Krieger Publishing company, Malabar, Florida

14. Hamina, M., Saranen, J. (1992): Spline Collocation Method for the Single Layer Heat Operator. Boundary Elements XIV, Sevilla, Proceedings, Vol. 1, 349-363

15. Hamina, M., Saranen, J. (1994): On the spline collocation method for the single-layer heat operator equation. Math. Comp. 62, 41-64

16. Hsiao, G.C., Saranen, J. (1989): Coercivity for the single layer heat operator. Technical report 89-2, Department of Mathematical Sciences, University of Delaware

17. Hsiao, G.C., Saranen, J. (1989): Boundary integral solution of some heat conduction problems. Proceedings of the International Conference on Integral Equations and Inverse Problems, Varna, September 18-23

18. Hsiao, G.C., Saranen, J. (1993): Boundary integral solution of the two-dimensional heat equation. Math. Methods Appl. Sci 16, 87-114

19. Hämäläinen, J. (1998): Spline collocation for the single layer heat equation. Ann. Acad. Sci. Fenn. Math. Dissertationes 113

20. Hämäläinen, J., Saranen, J. (1997): A collocation method for the single layer heat equation of the first kind. In: Constanda, C., Saranen, J., Seikkala, S. (eds.) Integral Methods in Science and Engineering, vol 2: Approximation Methods, Longman, Harlow, pp. 88-92.

21. Ladyženskaya, O.A., Solonnikov, V.A., Ural'ceva, N.N. (1968): Linear and Quasilinear Equations of Parabolic Type. Translations of the Mathematical Monographs 23, American Mathematical Society Providence, Rhode Island

22. Ch. Lubich, R. Schneider (1992): Time discretization of parabolic boundary integral equations. Numer. Math. 63, 455-481

23. Müntz, G.M. (1934): Zum dynamischen Wärmeleitungsproblem. Math. Z. 38, 323-338

24. Noon, J.P. (1988): The single layer heat potential and Galerkin boundary element methods for the heat equation. University of Maryland, Ph.D. Thesis

25. Onishi, K. (1981): Convergence in the boundary element method for the heat equation. TRU Math. 17, 213-225

26. Piriou, A. (1970): Une classe d'opérateurs pseudo-différentiels du type de Volterra. Ann. Inst. Fourier Grenoble 20, 77-94

27. Piriou, A. (1971): Problèmes aux limites généraux pour des opérateurs différentiels paraboliques dans un domaine borné. Ann. Inst. Fourier Grenoble 21, 59-78

28. Pogorzelski, W. (1951): Sur la solution de l'équation intégrale dans le problème de Fourier. Ann. Soc. Math. Polon. Ser. III 24, 56-64

29. Pogorzelski, W. (1956): Sur le problème de Fourier généralisé. Ann. Polon. Math. 3, 126-141

30. Pogorzelski, W. (1964): Integral Equations 1, Pergamon Press, Oxford 
31. Prössdorf, S., Schneider, R. (1991): A spline collocation method for multidimensional strongly elliptic pseudodifferential operators of order zero. Integral Equations Operator Theory 14, 399-435

32. Prössdorf, S., Schneider, R. (1992): Spline approximation methods for multidimensional periodic pseudodifferential equations. Integral Equations Operator Theory 15, 626-672

33. Saranen, J. (1988): The convergence of even degree spline collocation solution for potential problems in smooth domains of the plane. Numer. Math. 53, 499-512

34. Saranen, J., Wendland, W.L. (1985): On the asymptotic convergence of collocation methods with spline functions of even degree. Math. Comp. 45, 91-108 